(C) 2005 Ämerican Chemical Society, J. Org. Chem., Davis jo0402780 Supporting Info Page 1

Asymmetric Synthesis of $\beta$-Amino Carbonyl Compounds using $N$-Sulfinyl $\beta$-Amino

\title{
Weinreb Amides
}

Franklin A. Davis*, M. Brad Nolt, Yongzhong Wu, Kavirayani R. Prasad, Danyang Li, Bin Yang, Kerisha Bowen, Seung H. Lee, and John H. Eardley

Department of Chemistry, Temple University, Philadelphia, Pennsylvania 19122 fdavis@temple.edu

\section{Supporting Information Available}

\section{Contents}

\begin{tabular}{|c|c|c|c|c|c|c|c|c|}
\hline Page & $\mathrm{S} 2 \mathrm{C}$ & neral Exper & ntal I & rocedures & & & & \\
\hline Page & S3 & ${ }^{1} \mathrm{H}$ NMR & $2 d$ & & Page & S32 & ${ }^{1} \mathrm{H}$ NMR & 14 \\
\hline Page & S4 & ${ }^{13} \mathrm{C}$ NMR & $2 d$ & & Page & S33 & ${ }^{13} \mathrm{C}$ NMR & 14 \\
\hline Page & S5 & ${ }^{1} \mathrm{H}$ NMR & $\mathbf{3 b}$ & & Page & S34 & ${ }^{1} \mathrm{H}$ NMR & 15 \\
\hline Page & S6 & ${ }^{13}$ C NMR & $3 \mathbf{b}$ & & Page & S35 & ${ }^{13} \mathrm{C}$ NMR & 15 \\
\hline Page & S7 & ${ }^{1} \mathrm{H}$ NMR & $3 \mathbf{c}$ & & Page & S36. & 'H NMR & 16 \\
\hline Page & S8 & ${ }^{13} \mathrm{C} \cdot \mathrm{NMR}$ & $3 c$ & & Page & S37 & ${ }^{13} \mathrm{C}$ NMR & 16 \\
\hline Page & S9 & ${ }^{1} \mathrm{H}$ NMR & 3d & & & & & \\
\hline Page & S10 & ${ }^{13} \mathrm{C}$ NMR & 3d & & & & & \\
\hline Page & S11 & ${ }^{1} \mathrm{H}$ NMR & $3 e$ & & & & & \\
\hline Page & S12 & ${ }^{13} \mathrm{C}$ NMR & $3 \mathbf{e}$ & & & & & \\
\hline Page & S13 & ${ }^{1} \mathrm{H}$ NMR & $3 \mathbf{g}$ & & & & & \\
\hline Page & S14 & ${ }^{13} \mathrm{C}$ NMR & $3 \mathbf{g}$ & & & & & \\
\hline Page & S15 & ${ }^{1} \mathrm{H}$ NMR & $4 \mathbf{a}$ & - & & & & \\
\hline Page & S16 & ${ }^{13} \mathrm{C}$ NMR & $4 \mathbf{a}$ & & & & & \\
\hline Page & S17 & ${ }^{1} \mathrm{H}$ NMR & $5 b$ & & & & & \\
\hline Page & S18 & ${ }^{13} \mathrm{C}$ NMR & $5 b$ & & & & & \\
\hline Page & S19 & ${ }^{1} \mathrm{H}$ NMR & $5 c$ & & & & & \\
\hline Page & S20 & ${ }^{13} \mathrm{C}$ NMR & $5 c$ & & & & & \\
\hline Page & S21 & ${ }^{1} \mathrm{H}$ NMR & $7 b$ & & & & & \\
\hline Page & $\mathrm{S} 22$ & ${ }^{13} \mathrm{C}$ NMR & $7 b$ & & & & & \\
\hline Page & S23 & ${ }^{1} \mathrm{H}$ NMR & $\mathbf{8 b}$ & & & & & \\
\hline Page & S24 & ${ }^{13} \mathrm{C}$ NMR & $\mathbf{8 b}$ & & & & & \\
\hline Page & S25 & ${ }^{1} \mathrm{H}$ NMR & $10 b$ & & & & & \\
\hline Page & S26 & ${ }^{13} \mathrm{C}$ NMR & $10 b$ & & & & & \\
\hline Page & S27 & ${ }^{1} \mathrm{H}$ NMR & 11 & & & & & \\
\hline Page & S28 & ${ }^{13} \mathrm{C} \cdot \mathrm{NMR}$ & 11 & & & & & \\
\hline Page & S29. & ${ }^{1} \mathrm{H}$ NMR & 13 & & & & & \\
\hline Page & $\mathrm{S} 30$ & ${ }^{13} \mathrm{C}$ NMR & 13 & & & & & \\
\hline Page & S31 & ${ }^{31}$ P NMR & 13 & & & & & \\
\hline
\end{tabular}


(C) 2005 American Chemical Society, J. Org. Chem., Davis jo0402780 Supporting Info Page 2

\section{Experimental}

General Procedures. Column chromatography was performed on silica gel, Merck

grade 60 (230-400 mesh). TLC plates were visualized with UV, in an iodine chamber, or with phosphomolybdic acid, unless noted otherwise. Unless stated otherwise, all reagents were purchased from commercial sources and used without additional purification. 
(1) 2005 American Chemical Society, J. Org. Chem., Davis jo0402780 Supporting Info Page 3

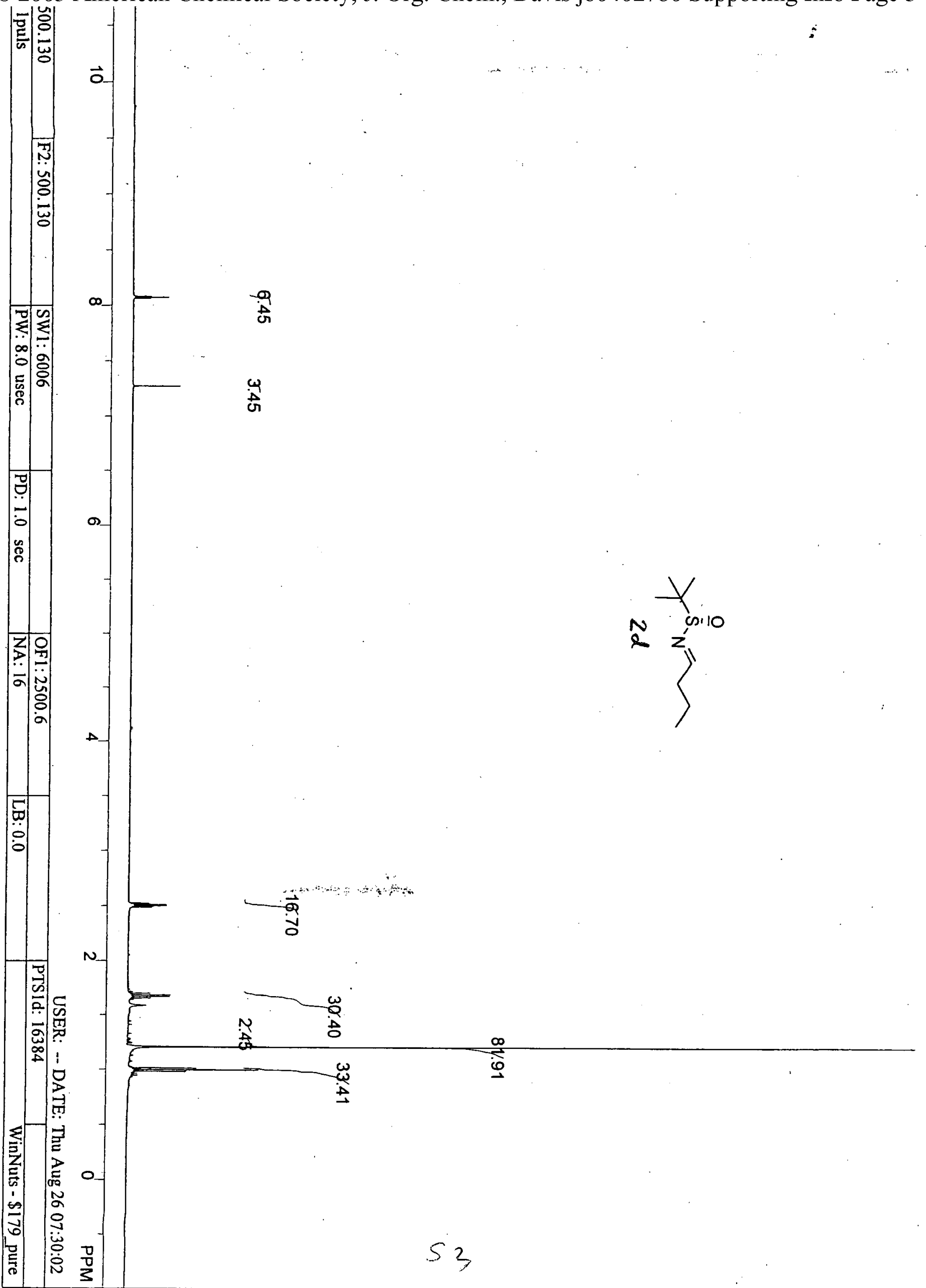


(C) 2005 American Chemical Society, J. Org. Chem., Davis jo0402780 Supporting Info Page 4

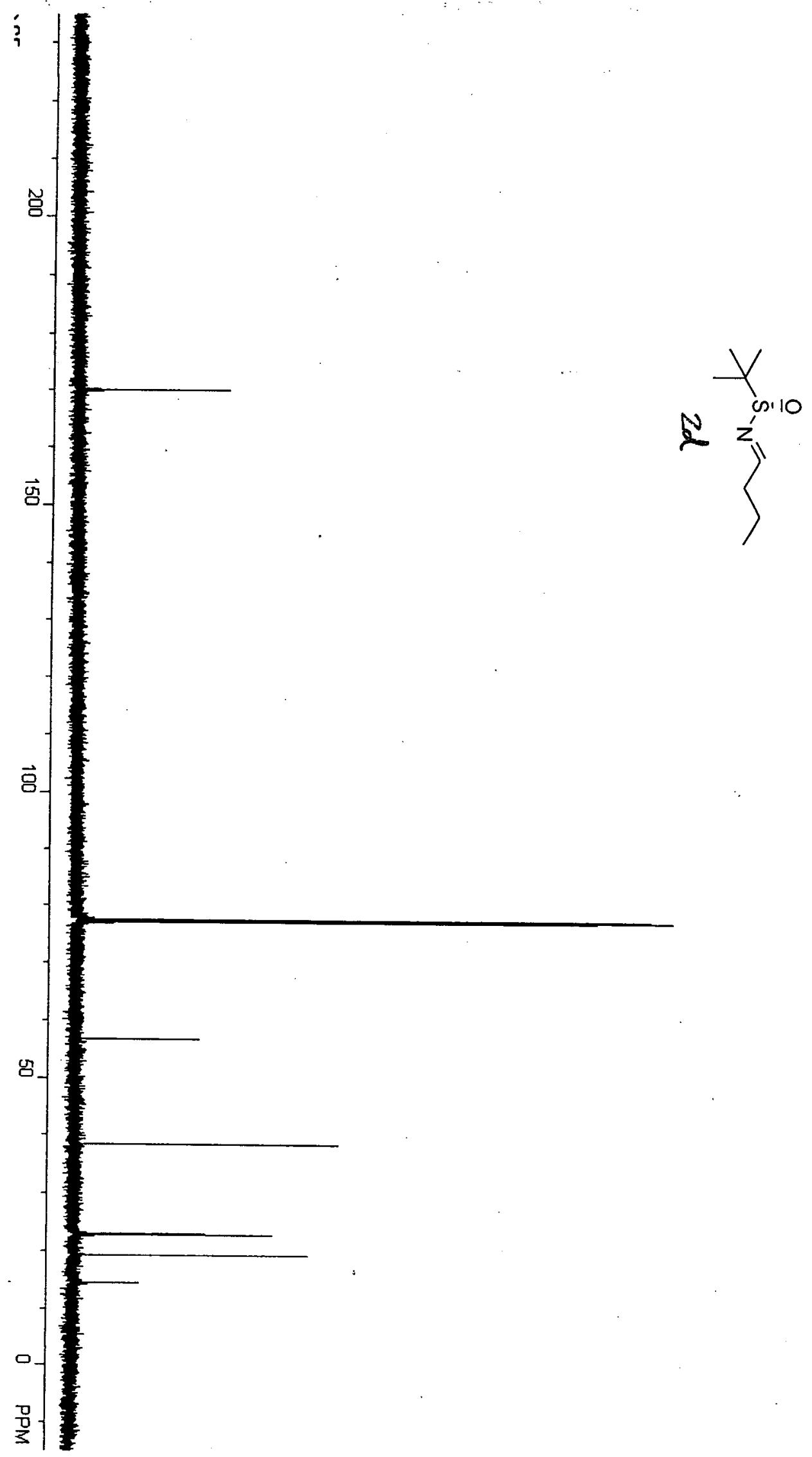


(C) 2005 American Chemical Society, J. Org. Chem., Davis jo0402780 Supporting Info Page 5
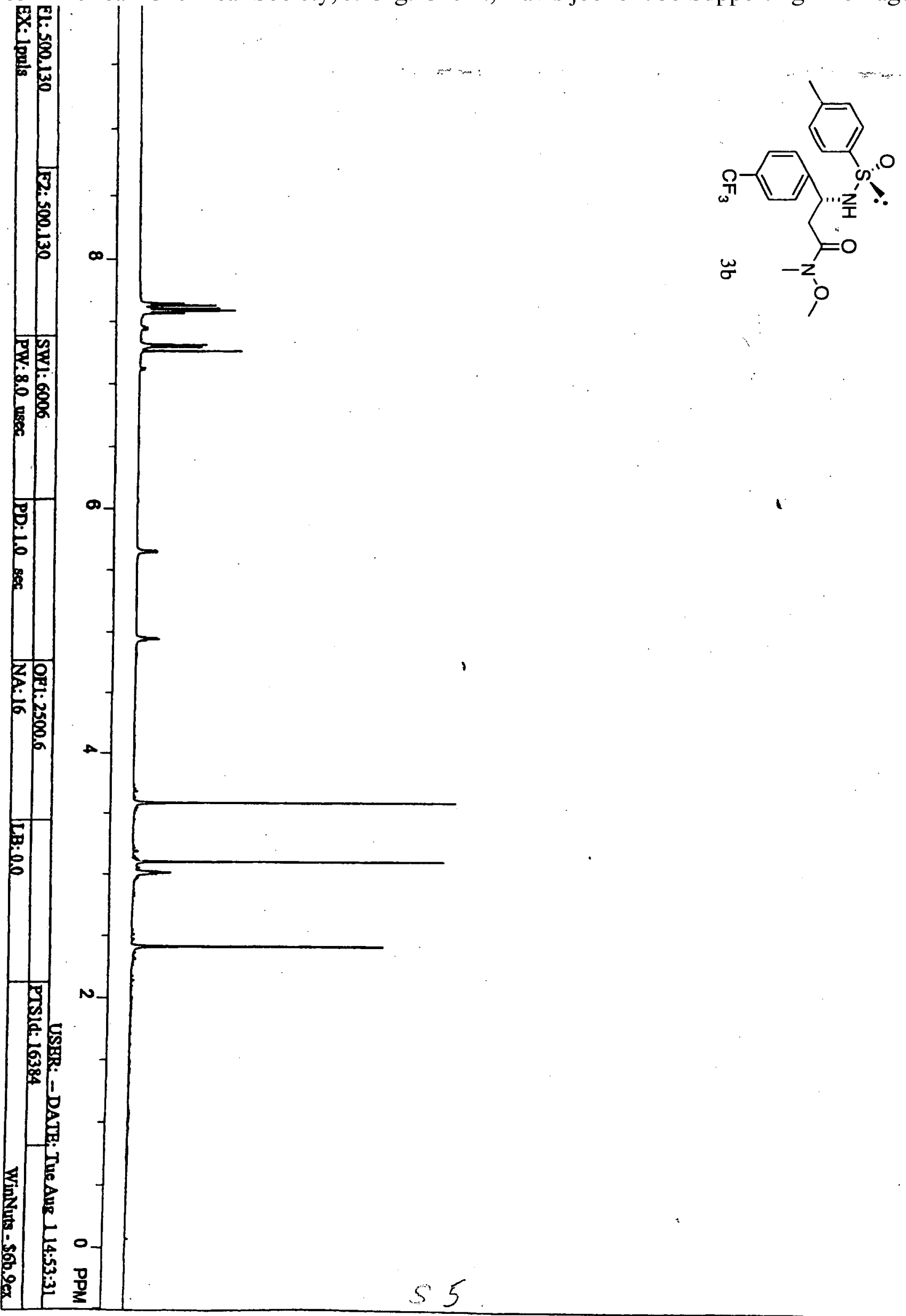
(C) 2005 American Chemical Society, J. Org. Chem., Davis jo0402780 Supporting Info Page 6

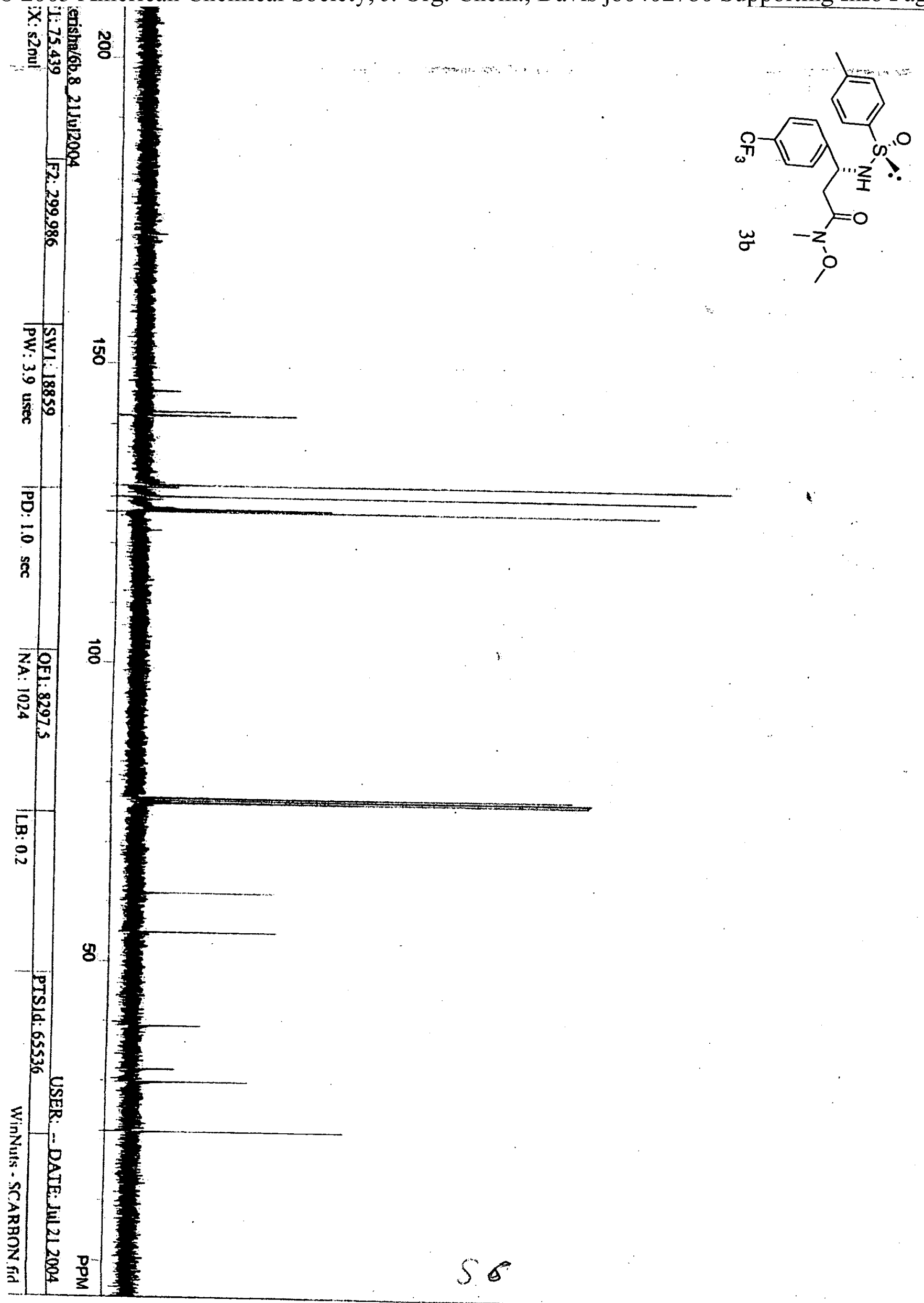


(C) 2005 American Chemical Society, J. Org. Chem., Davis jo0402780 Supporting Info Page 7

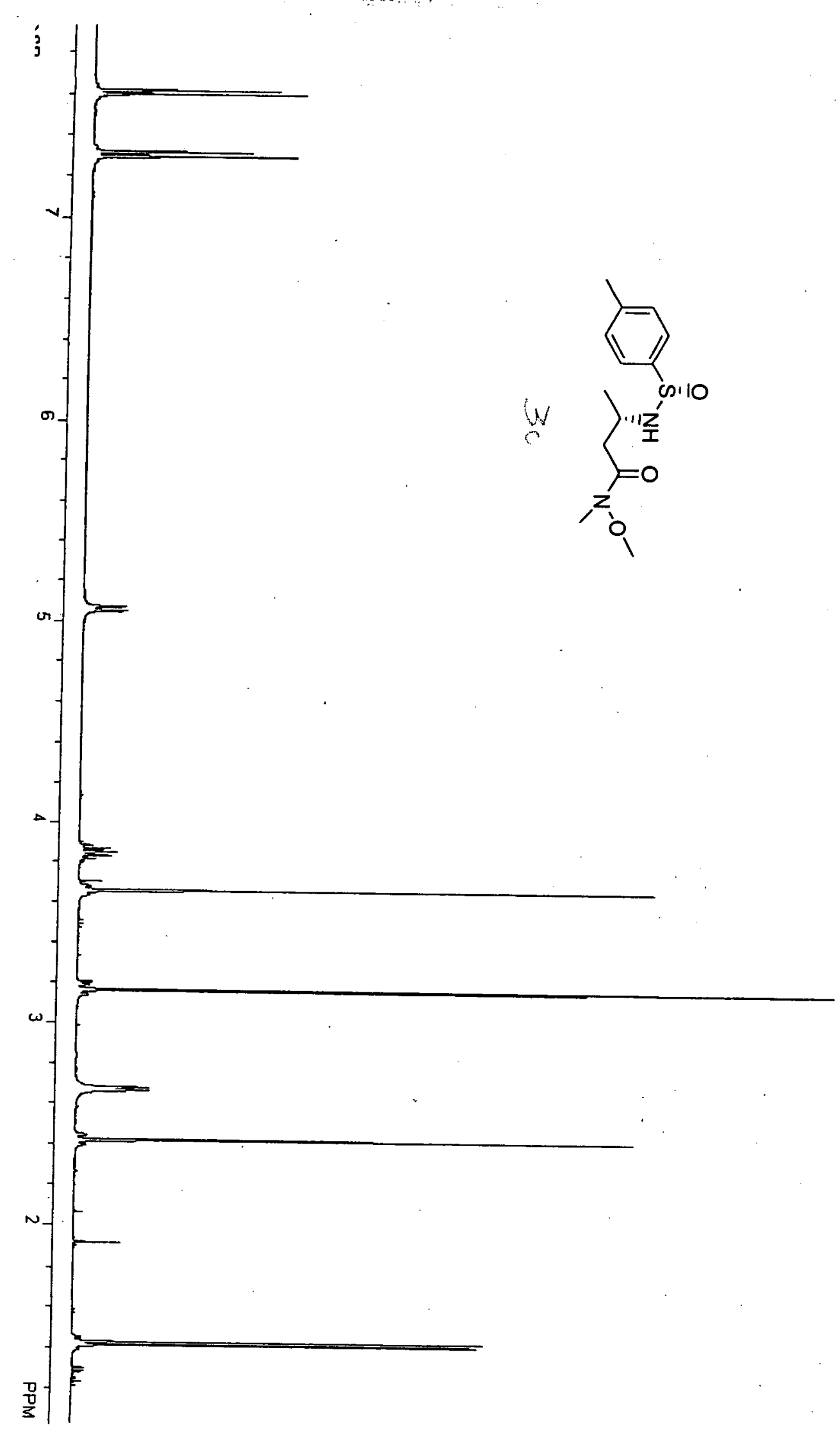

57 
(C) 2005 American Chemical Society, J. Org. Chem., Davis jo0402780 Supporting Info Page 8

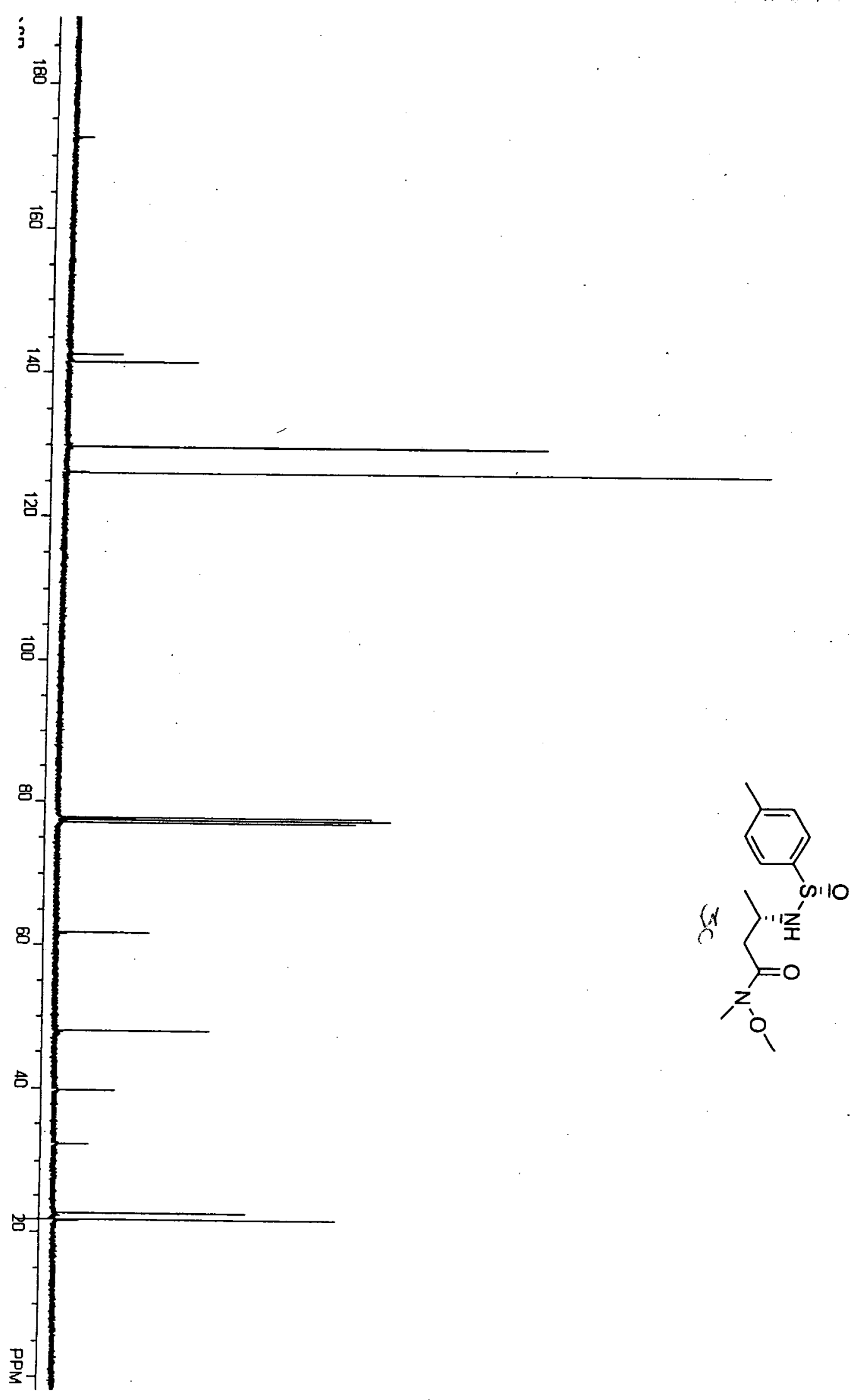


(C) 2005 American Chemical Society, J. Org. Chem., Davis jo0402780 Supporting Info Page 9

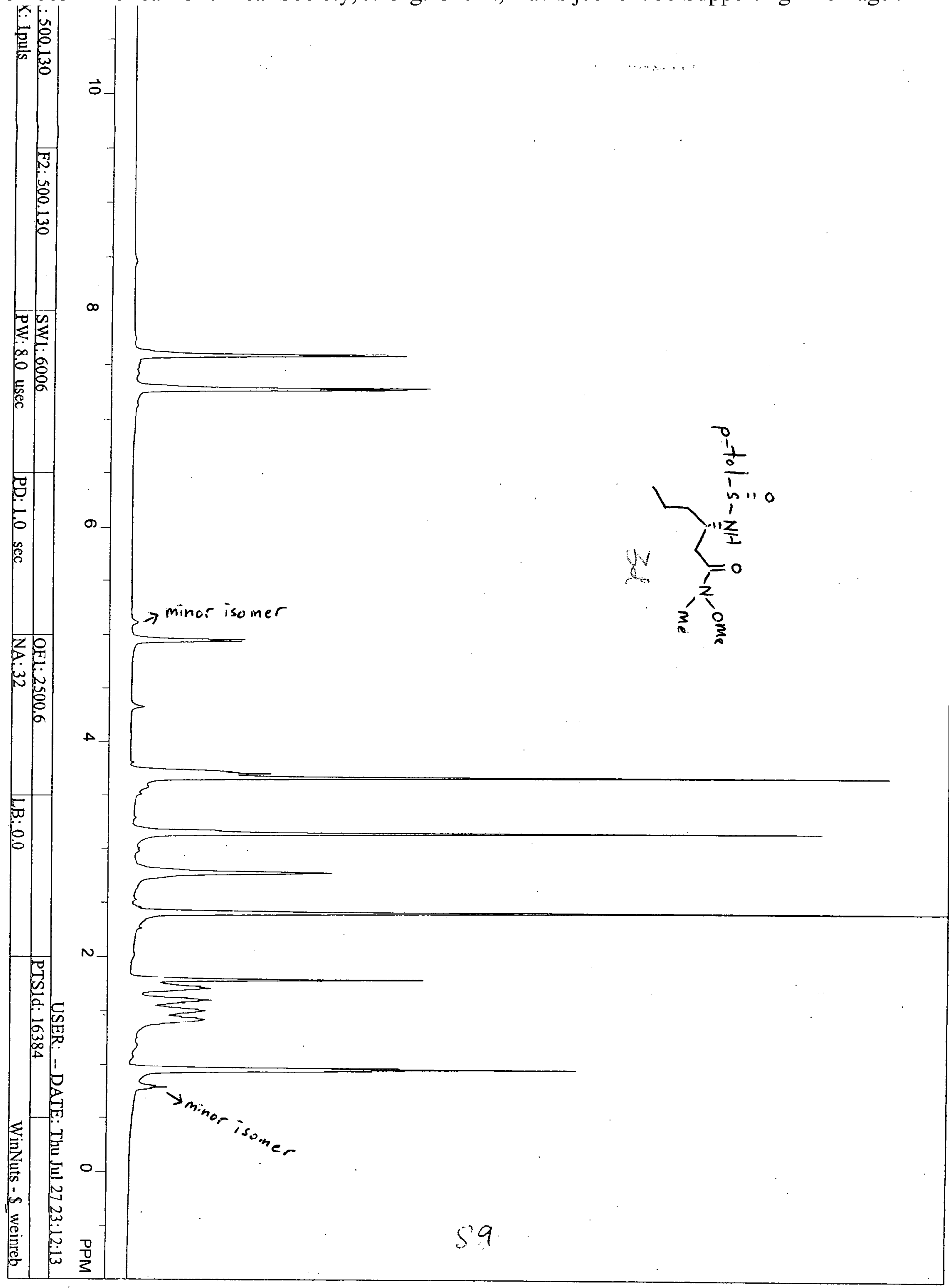


(c) 2005 American Chemical Society, J. Org. Chem., Davis jo0402780 Supporting Info Page 10
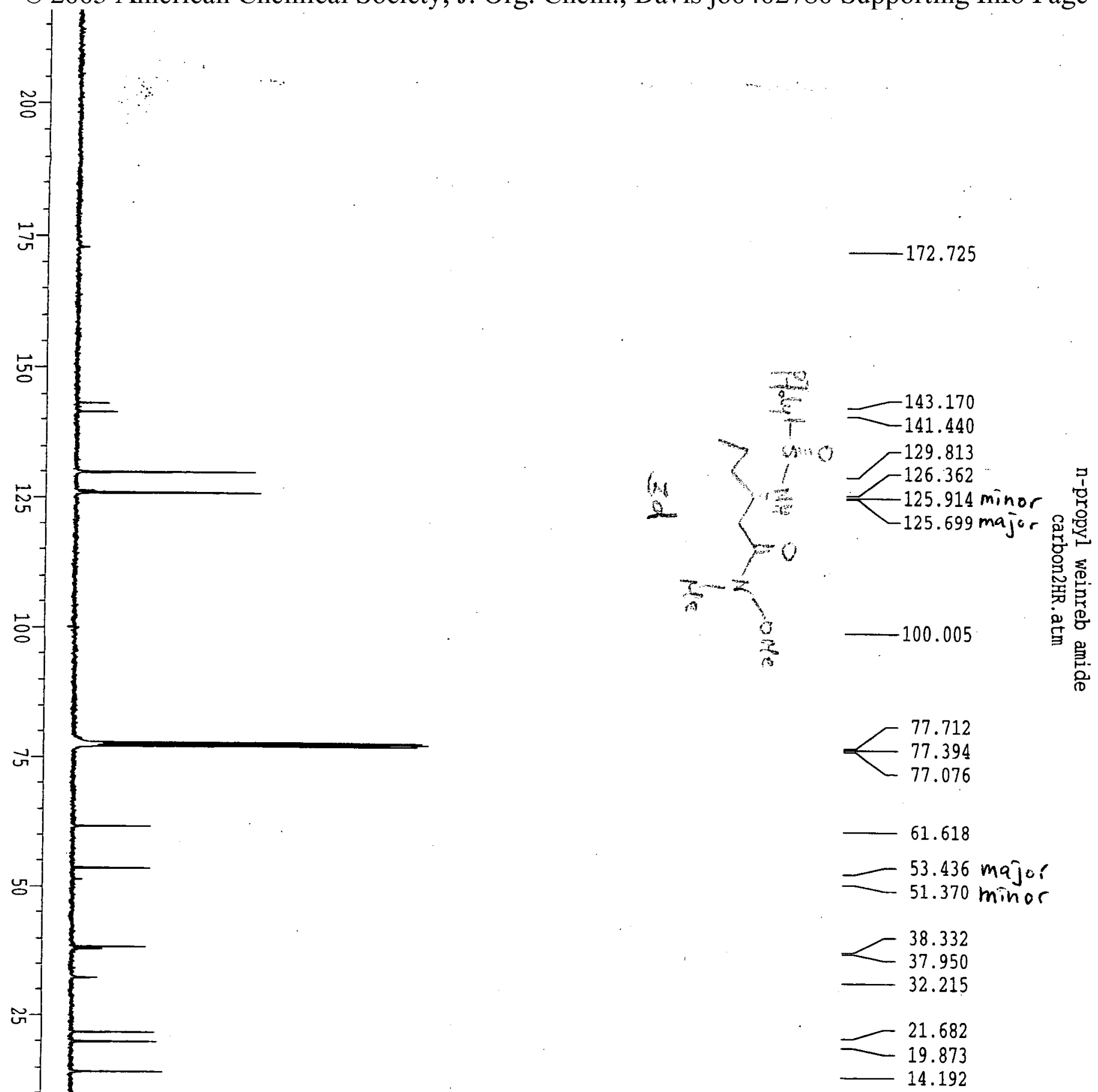

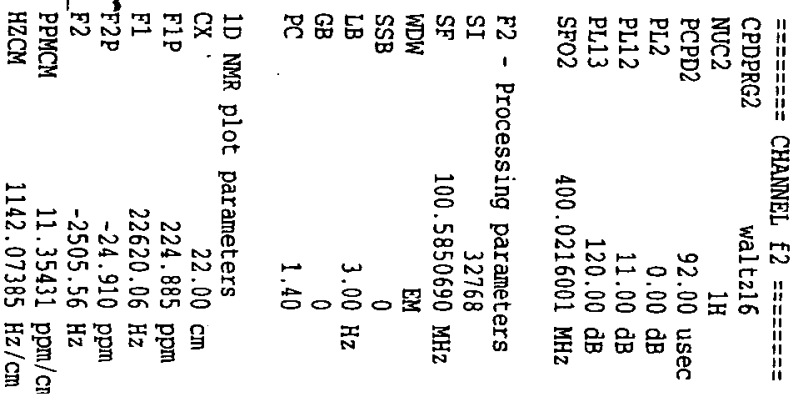

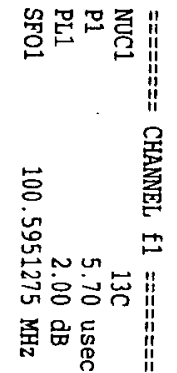

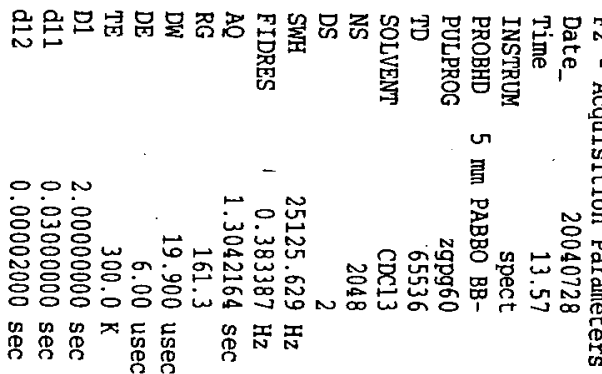

罗署焉点

510 
(C) 2005 American Chemical Society, J. Org. Chem., Davis jo0402780 Supporting Info Page 11
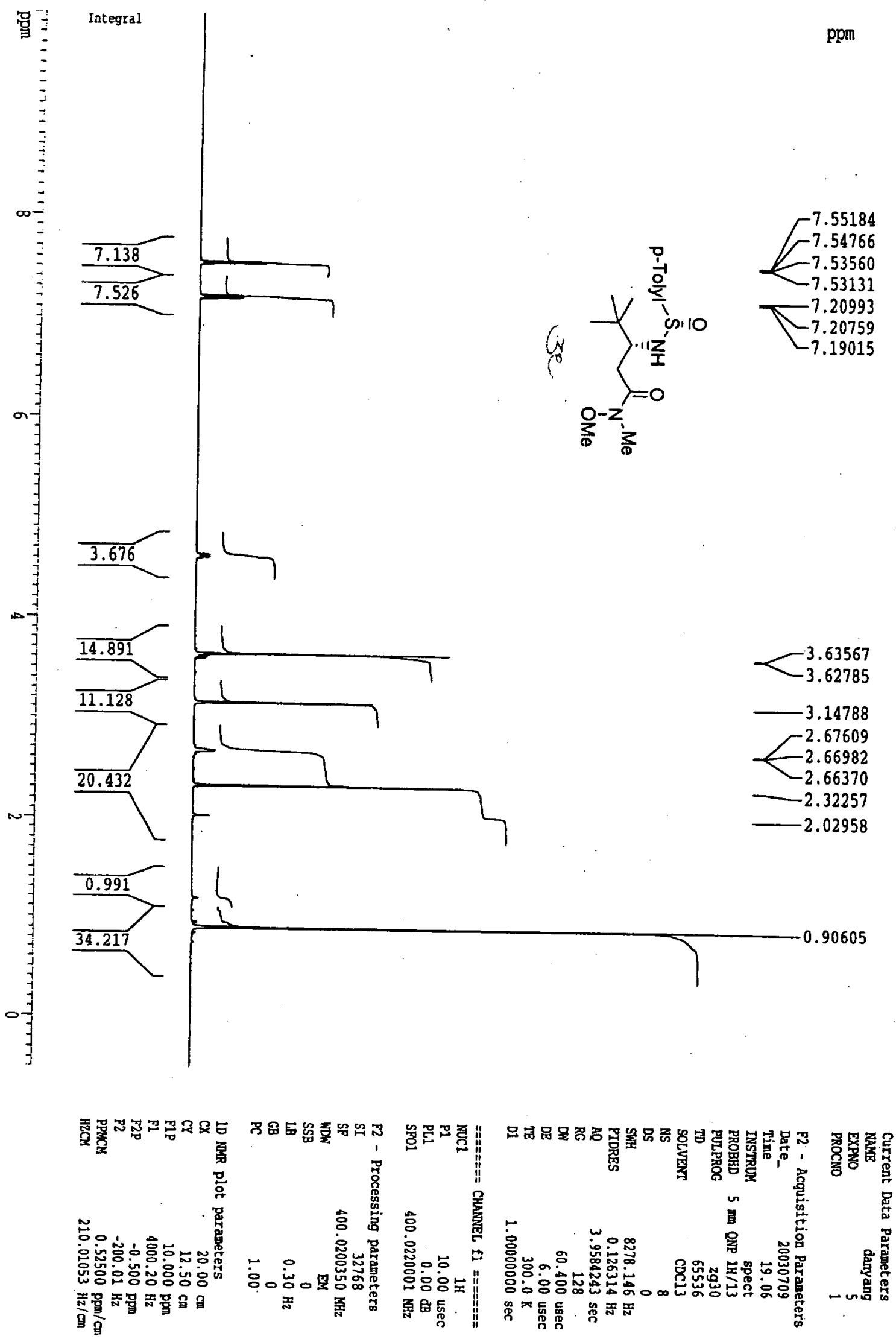
(C) 2005 American Chemical Society, J. Org. Chem., Davis jo0402780 Supporting Info Page 12
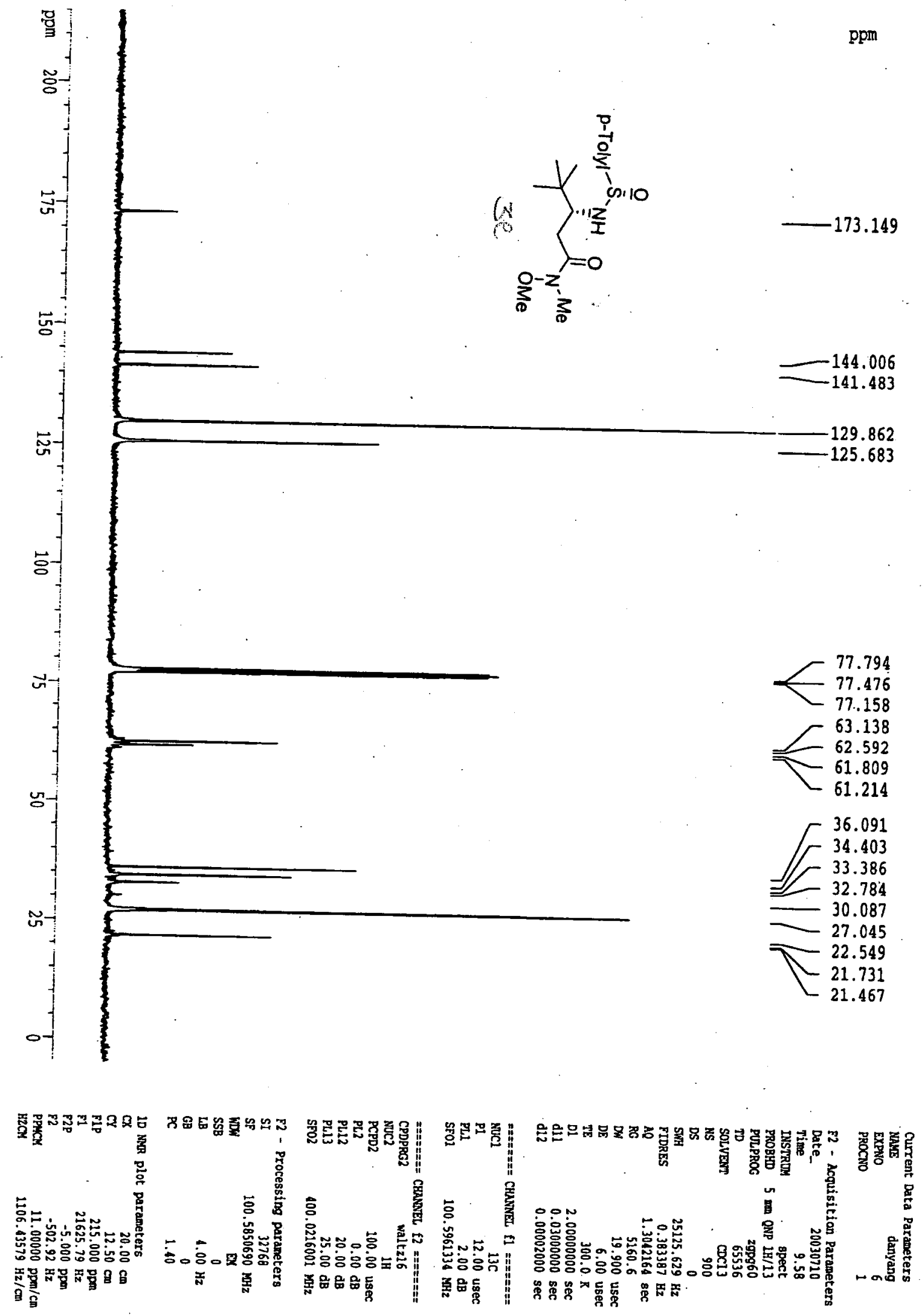


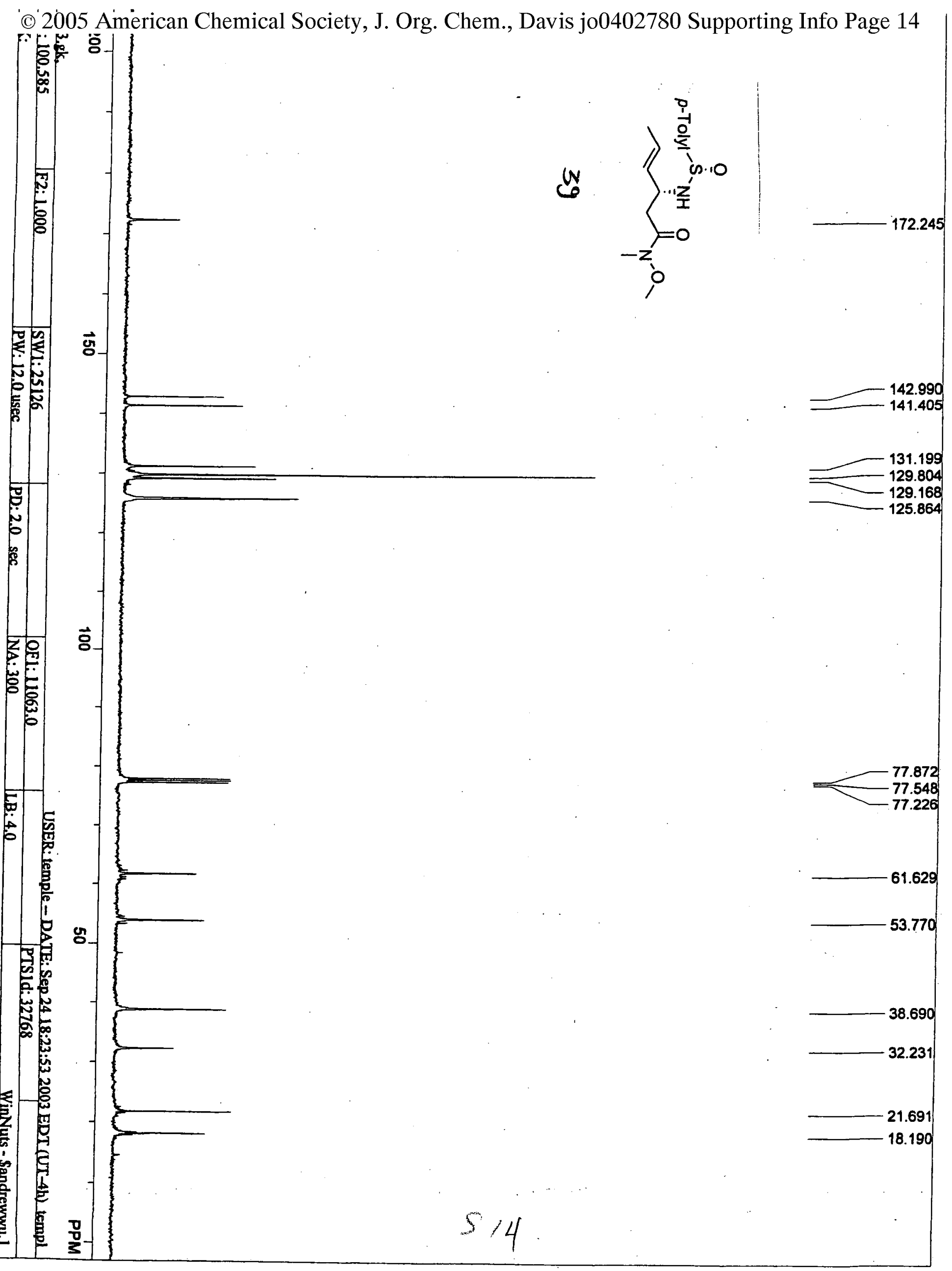


(1) 2005 American Chemical Society, J. Org. Chem., Davis jo0402780 Supporting Info Page 15

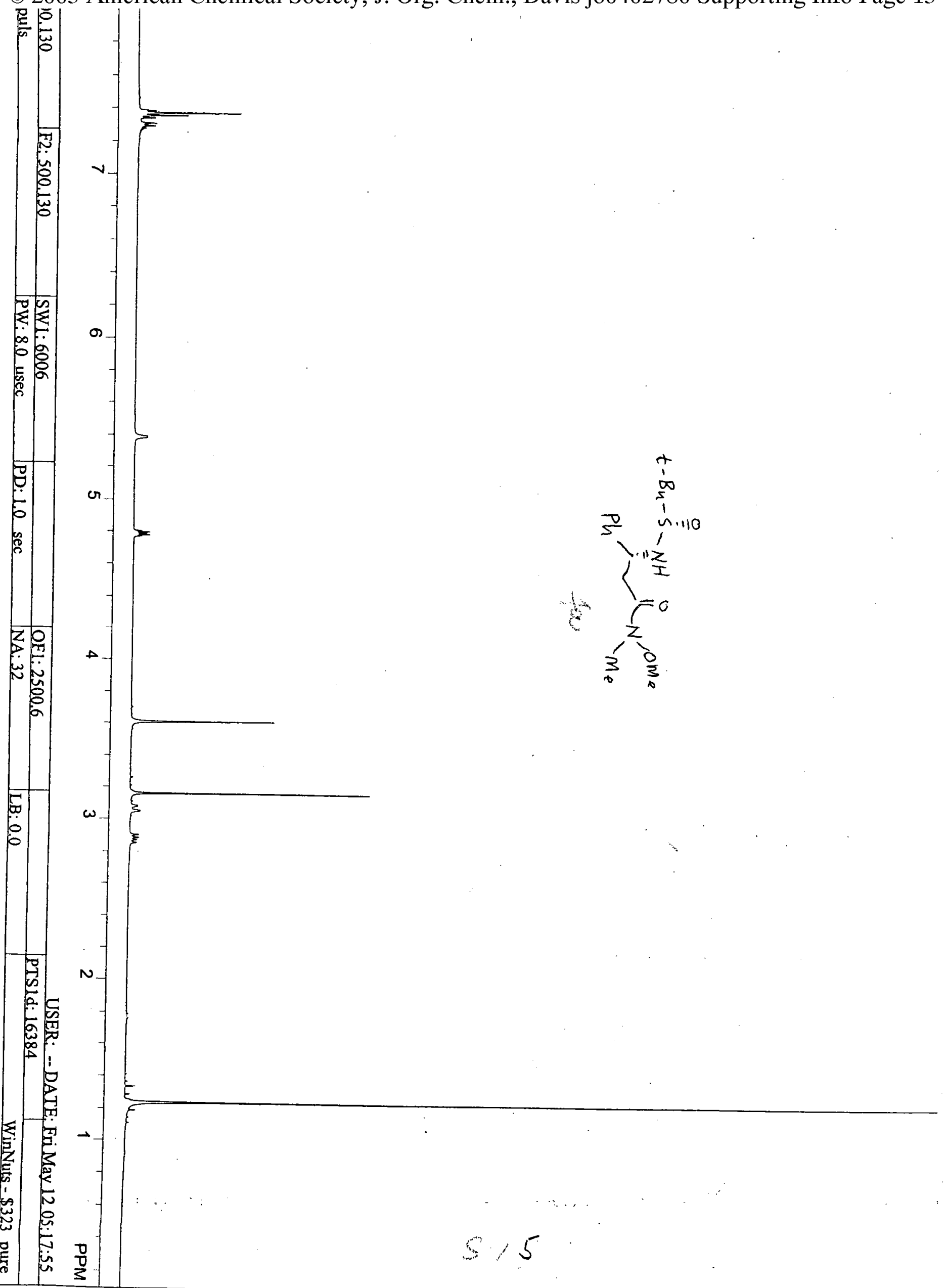


$\rightarrow 9$

(C) 2005 American Chemical Society, J. Org. Chem., Davis jo0402780 Supporting Info Page 16

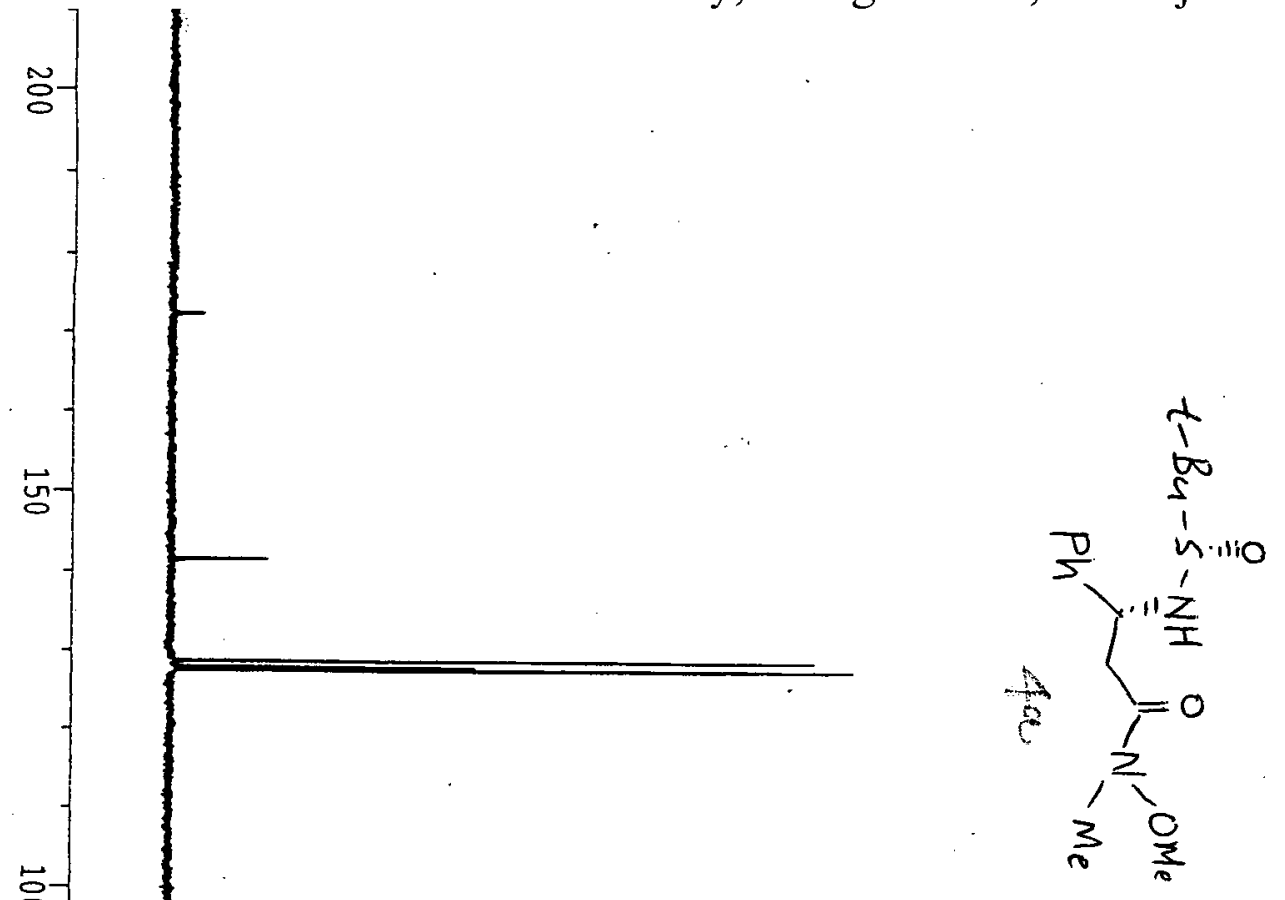

$-172.366$

8

77.694

77.582

77.377

77.059

61.731

55.995

55.890

39.464

32.322

23.109

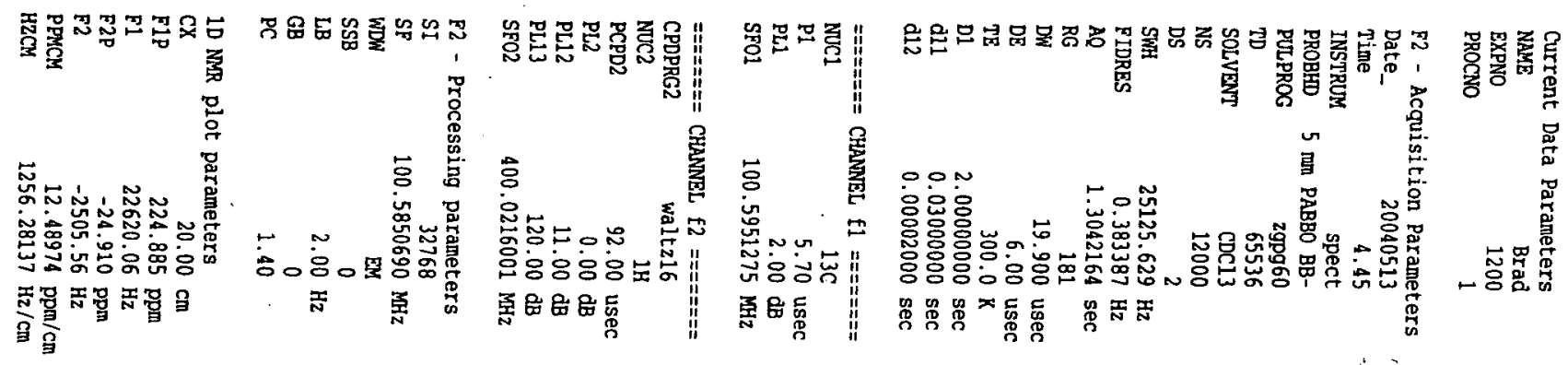


(C) 2005 American Chemical Society, J. Org. Chem., Davis jo0402780 Supporting Info Page 17

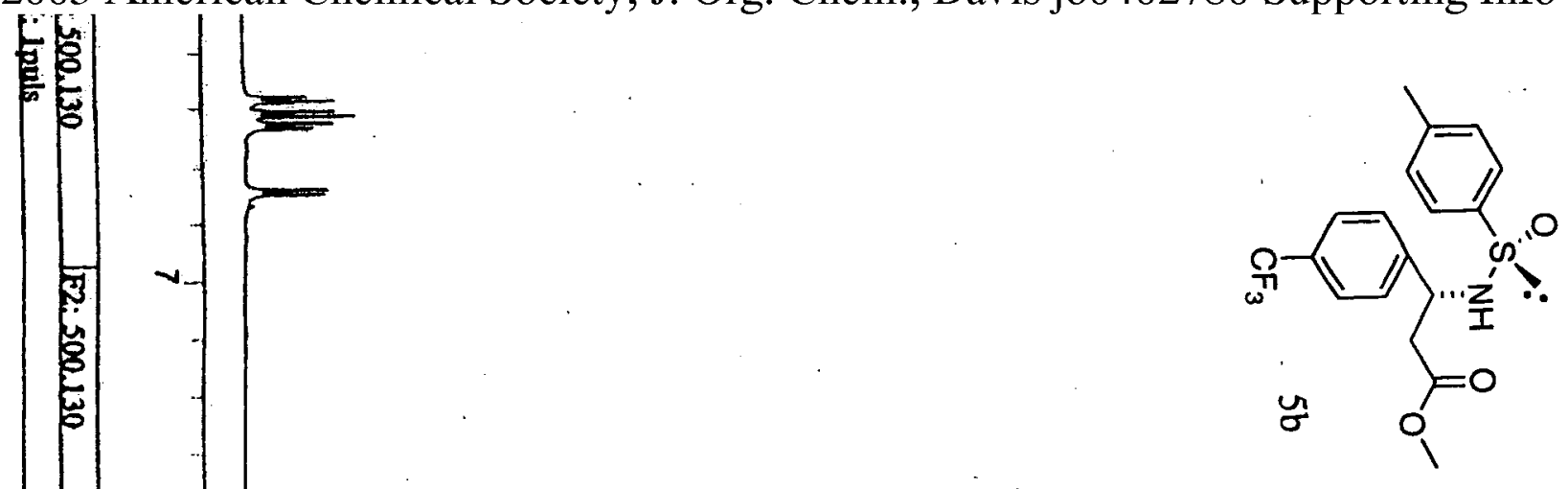

8

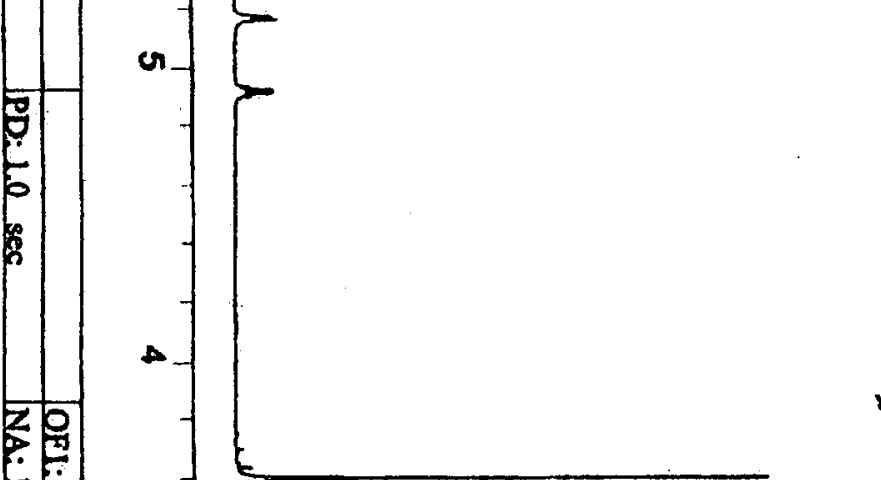

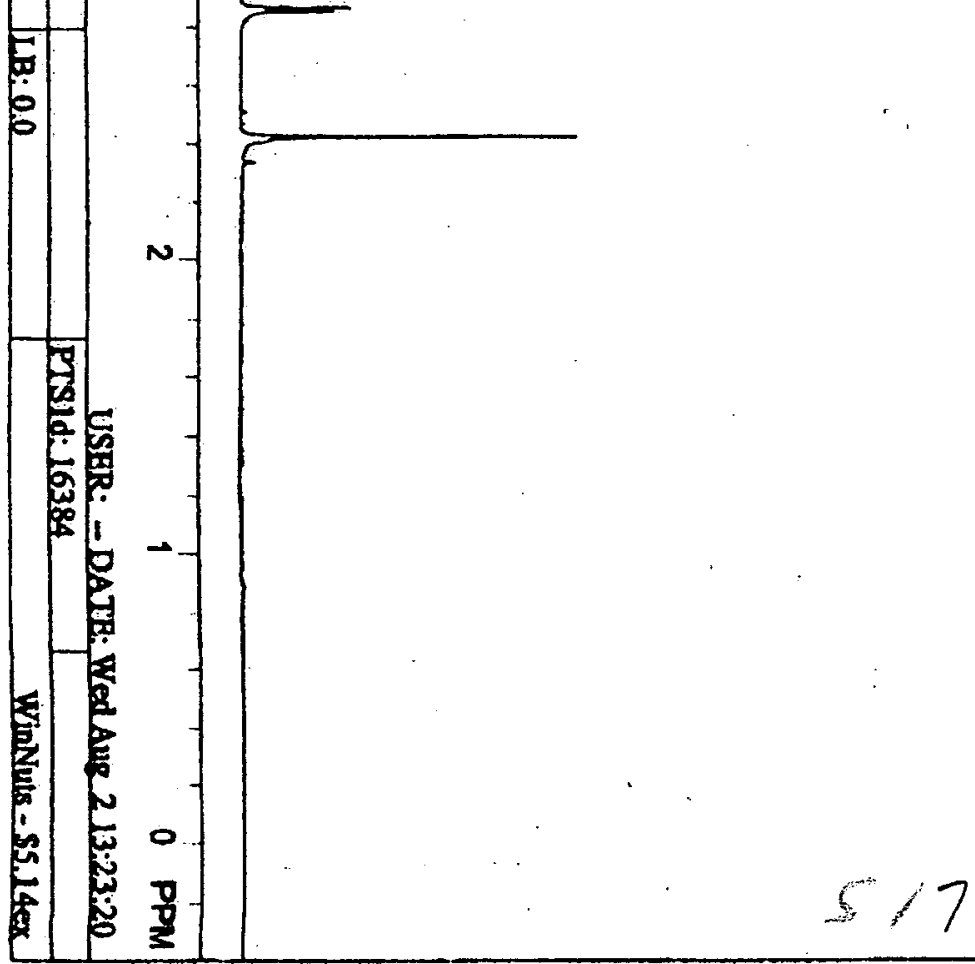


(C) 2005 American Chemical Society, J. Org. Chem., Davis jo0402780 Supporting Info Page 18

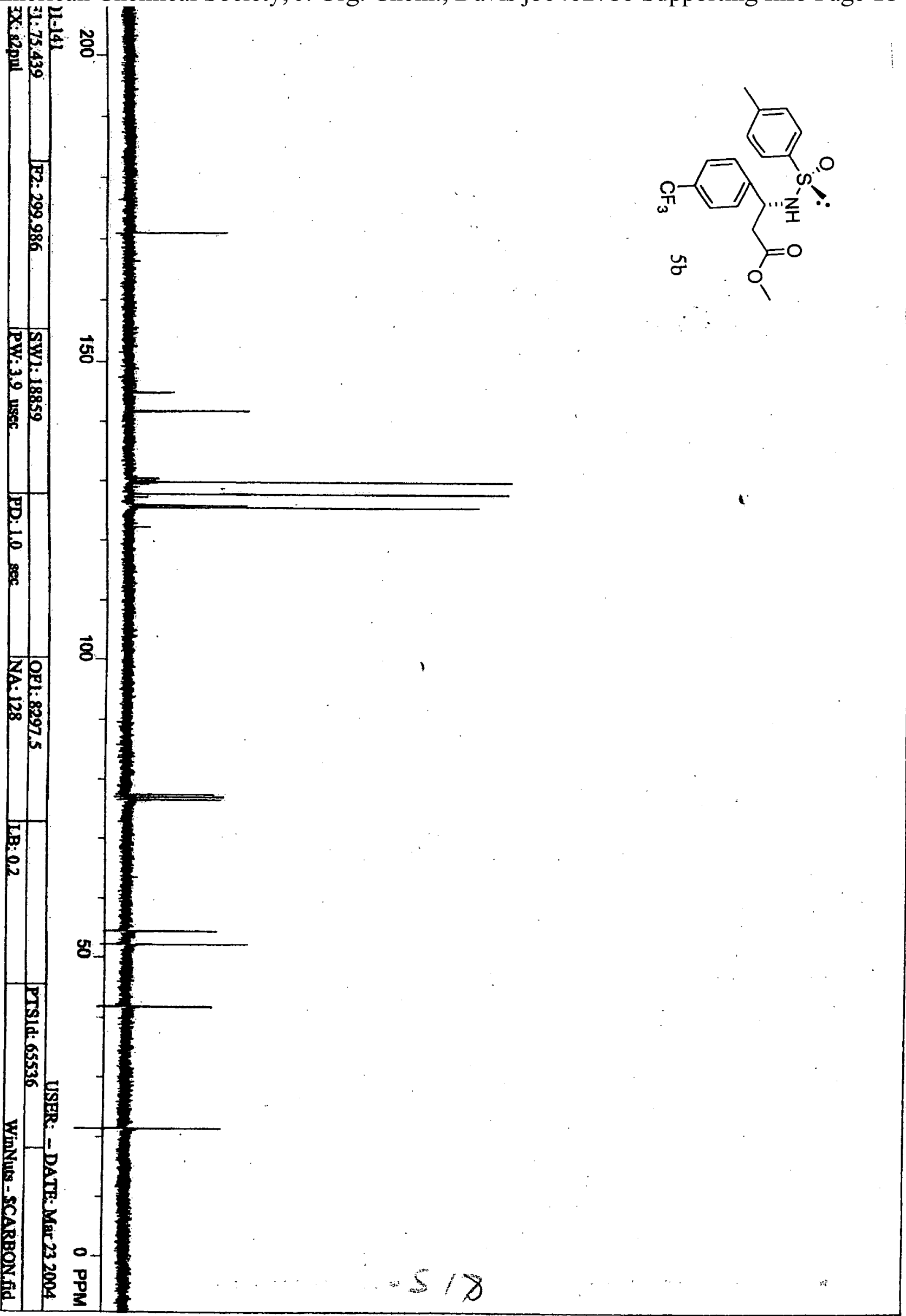


(C) 2005 American Chemical Society, J. Org. Chem., Davis jo0402780 Supporting Info Page 19

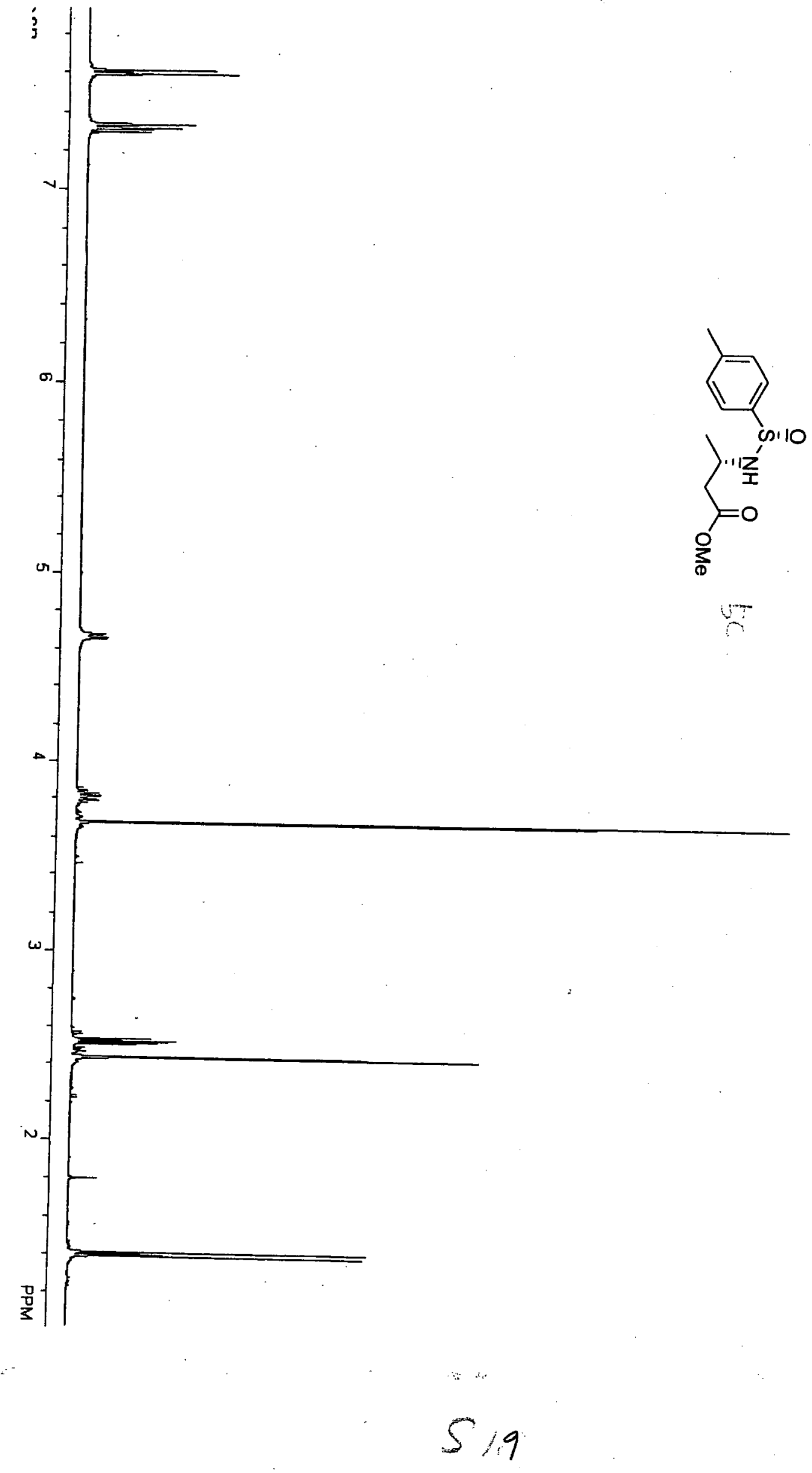


(C) 2005 American Chemical Society, J. Org. Chem., Davis jo0402780 Supporting Info Page 20

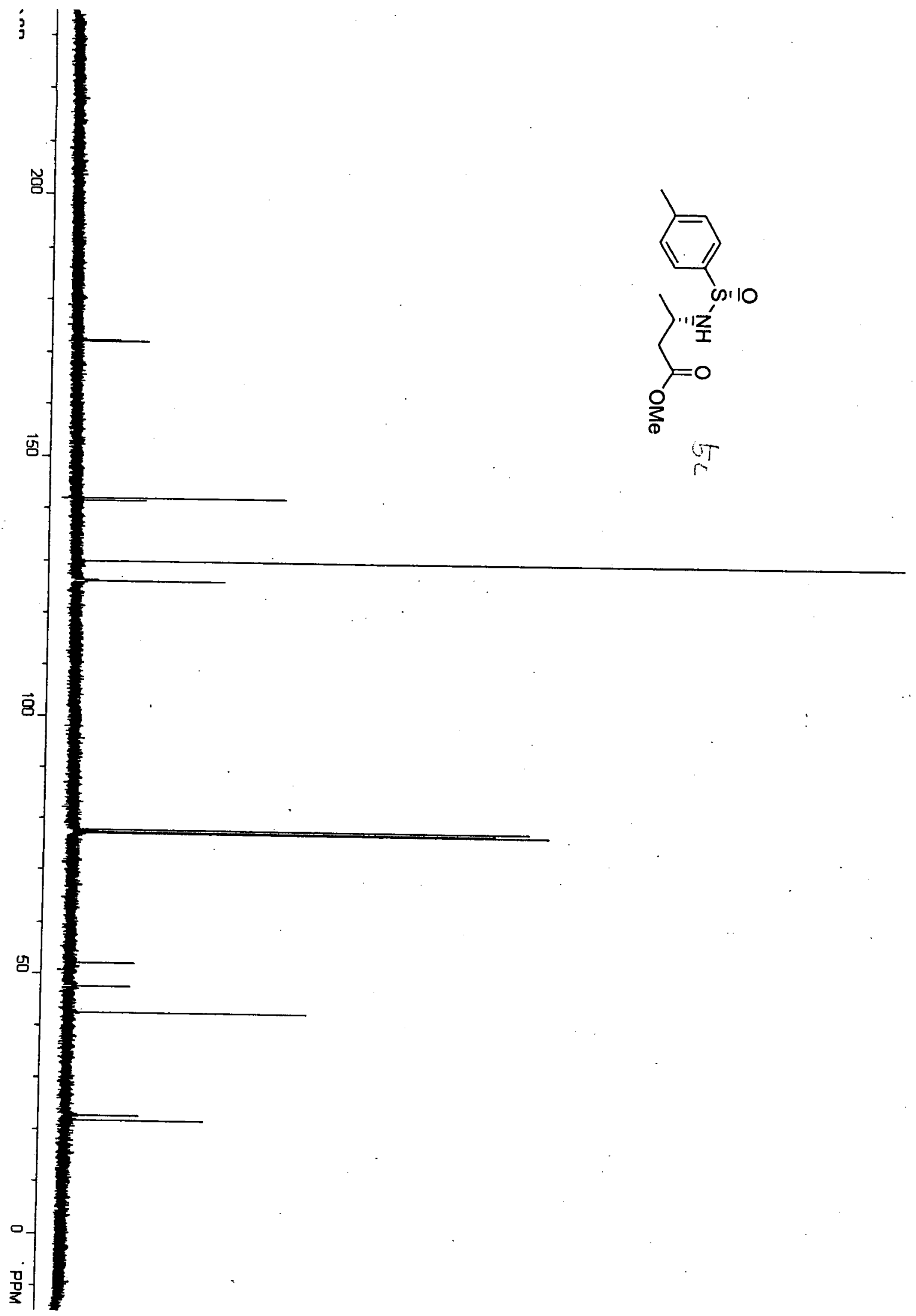


(C) 2005 American Chemical Society, J. Org. Chem., Davis jo0402780 Supporting Info Page 21

0

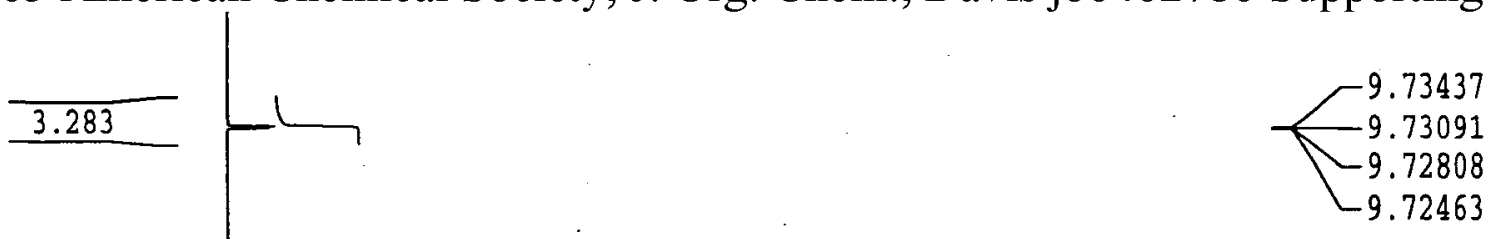

$\infty$
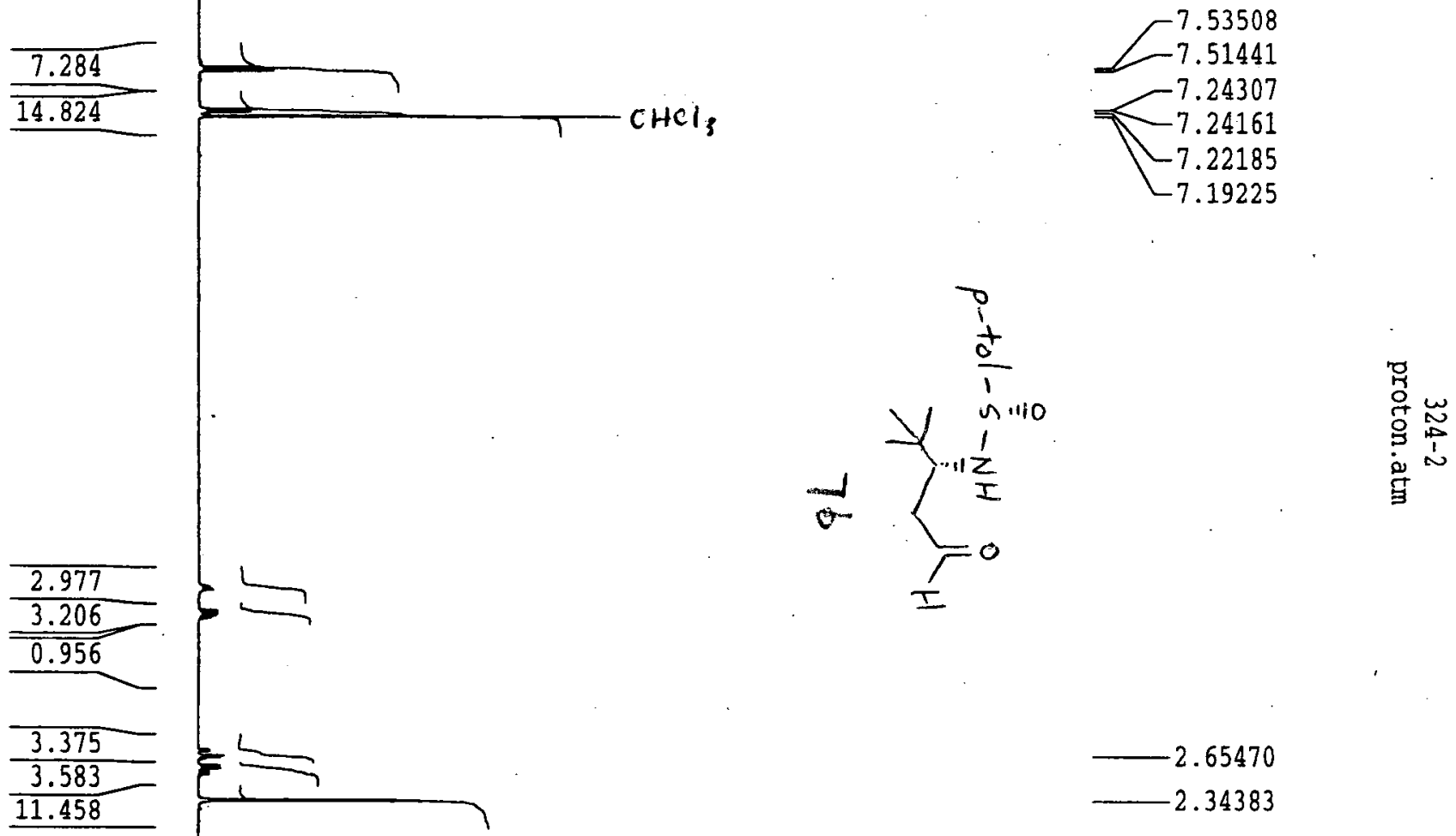

.65470

2.34383

\begin{tabular}{c}
$\frac{11.360}{1.457}$ \\
$\frac{-0.150}{35.830}$ \\
\hline-0.008 \\
\hline 0.564 \\
\hline
\end{tabular}

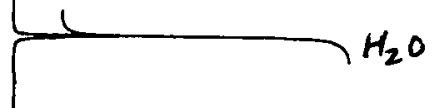

1.50905

0.90589
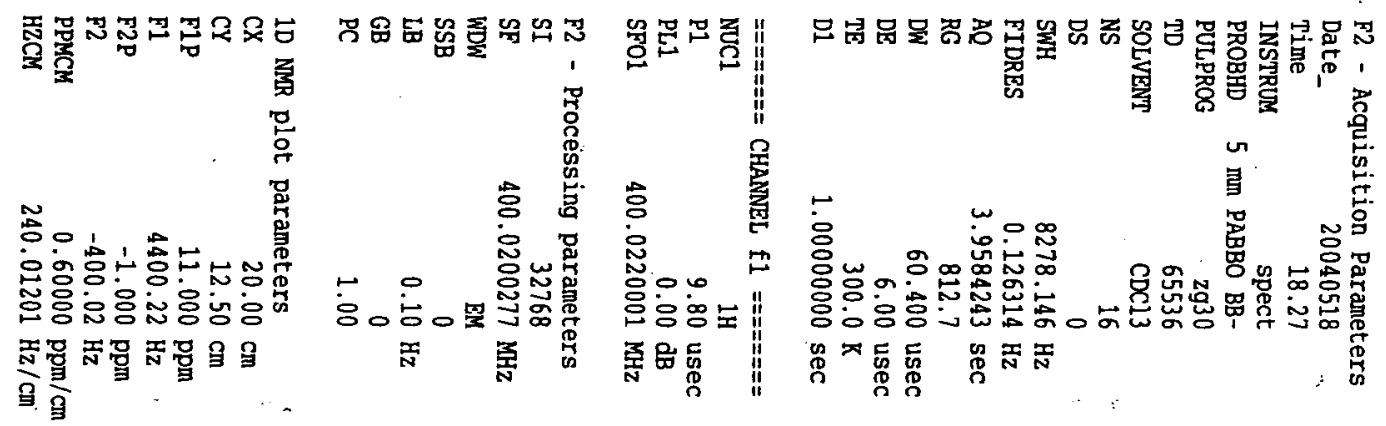

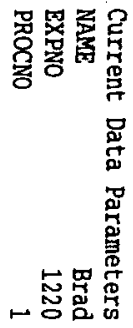

$$
521
$$




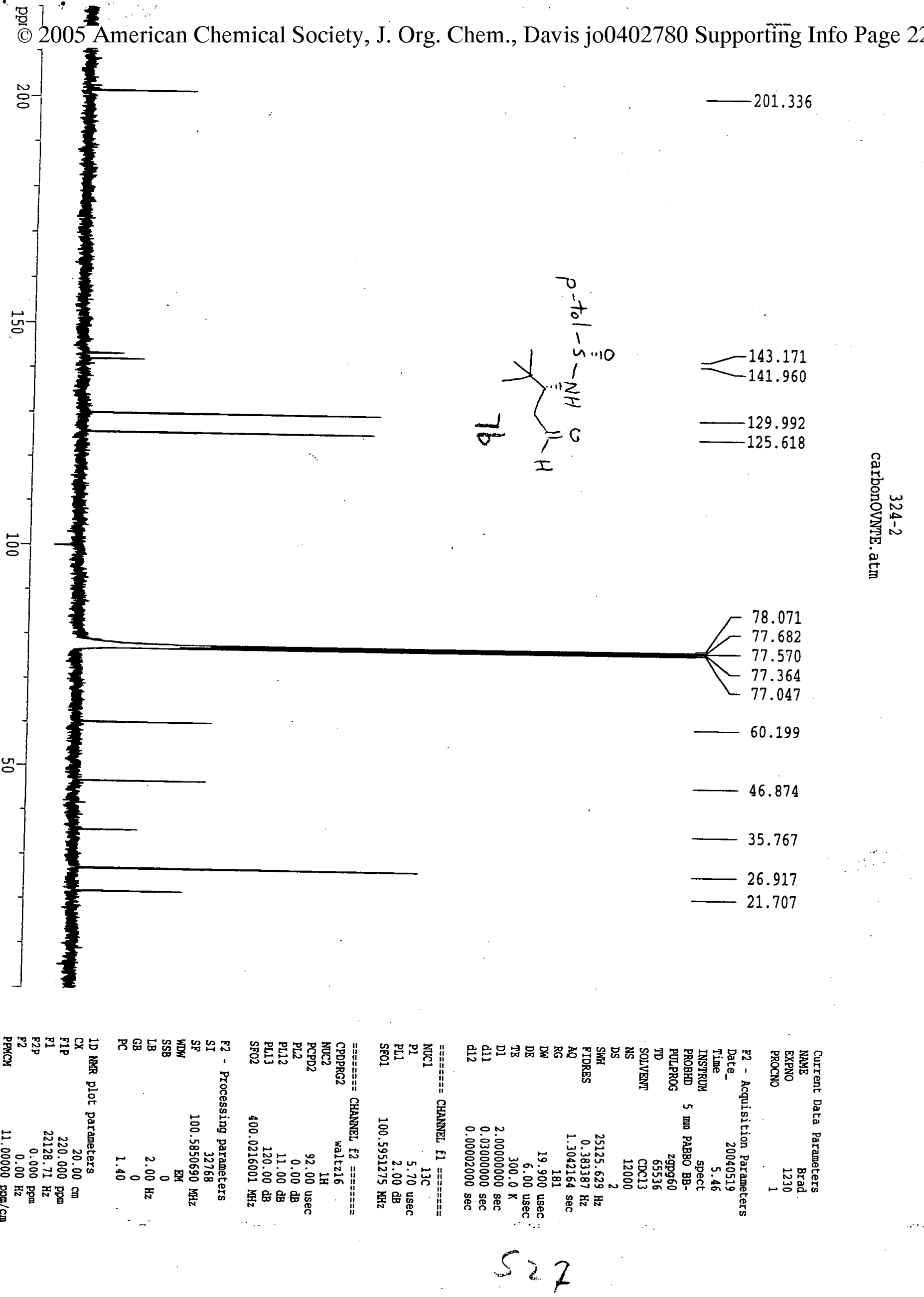


(C) 2005 American Chemical Society, J. Org. Chem., Davis jo0402780 Supporting Info Page 23

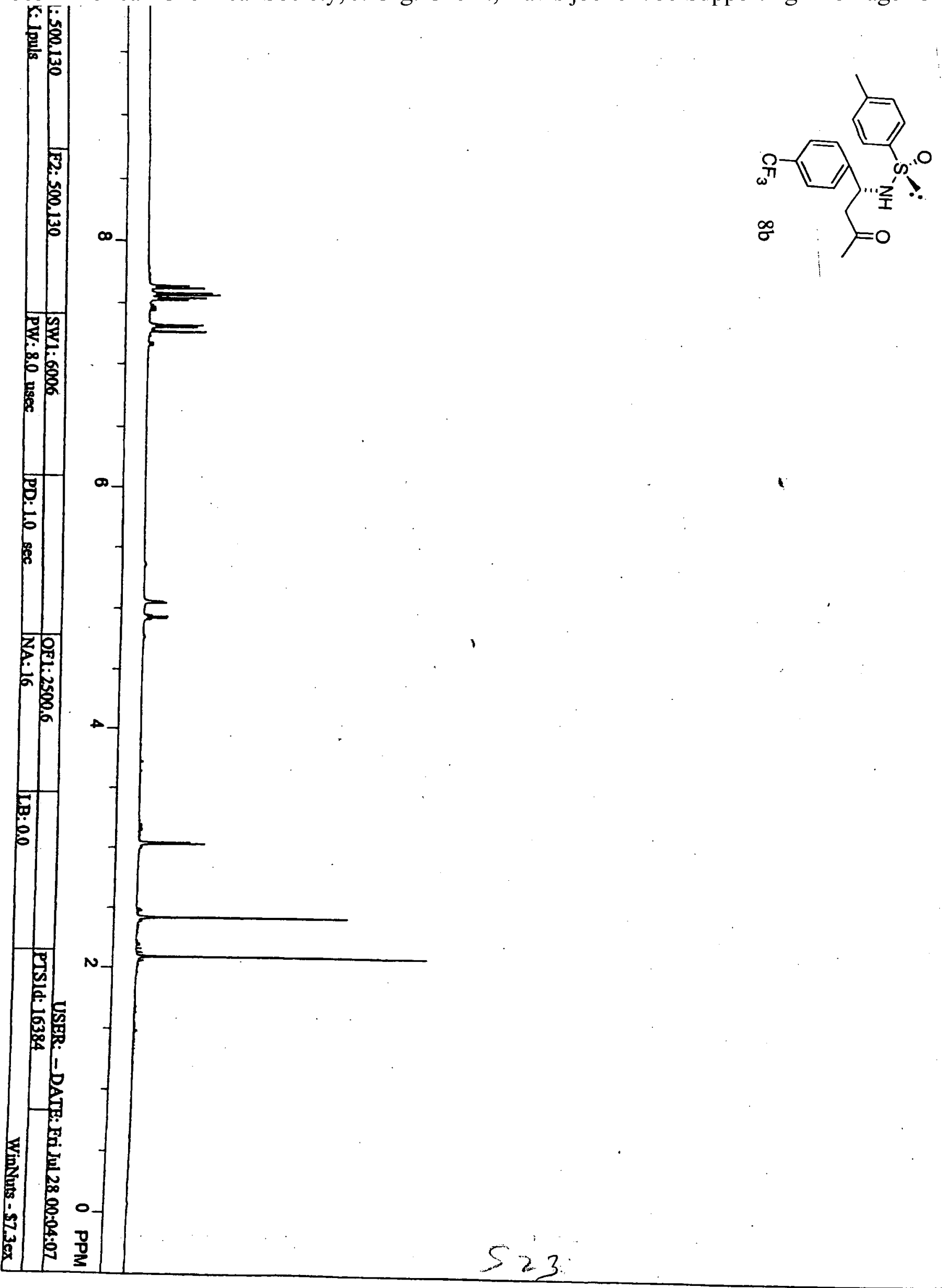




$$
F
$$


(C) 2005 American Chemical Society, J. Org. Chem., Davis jo0402780 Supporting Info Page 25

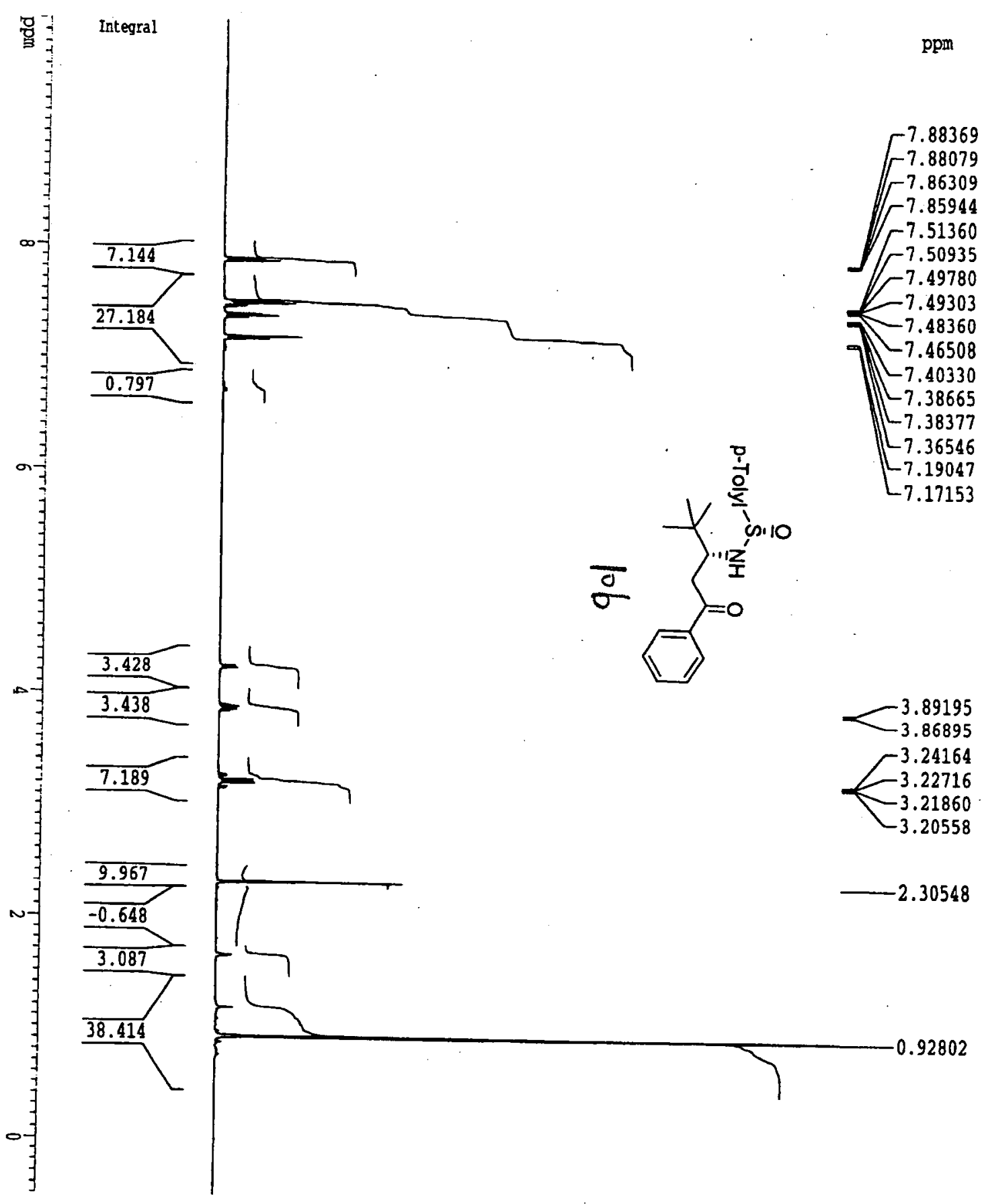

圆察

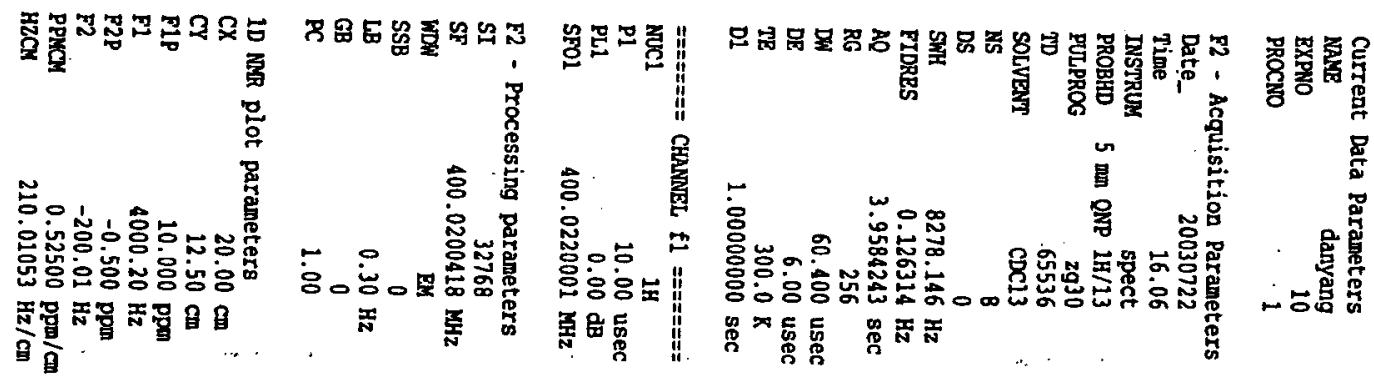


(C) 2005 American Chemical Society, J. Org. Chem., Davis jo0402780 Supporting Info Page 26
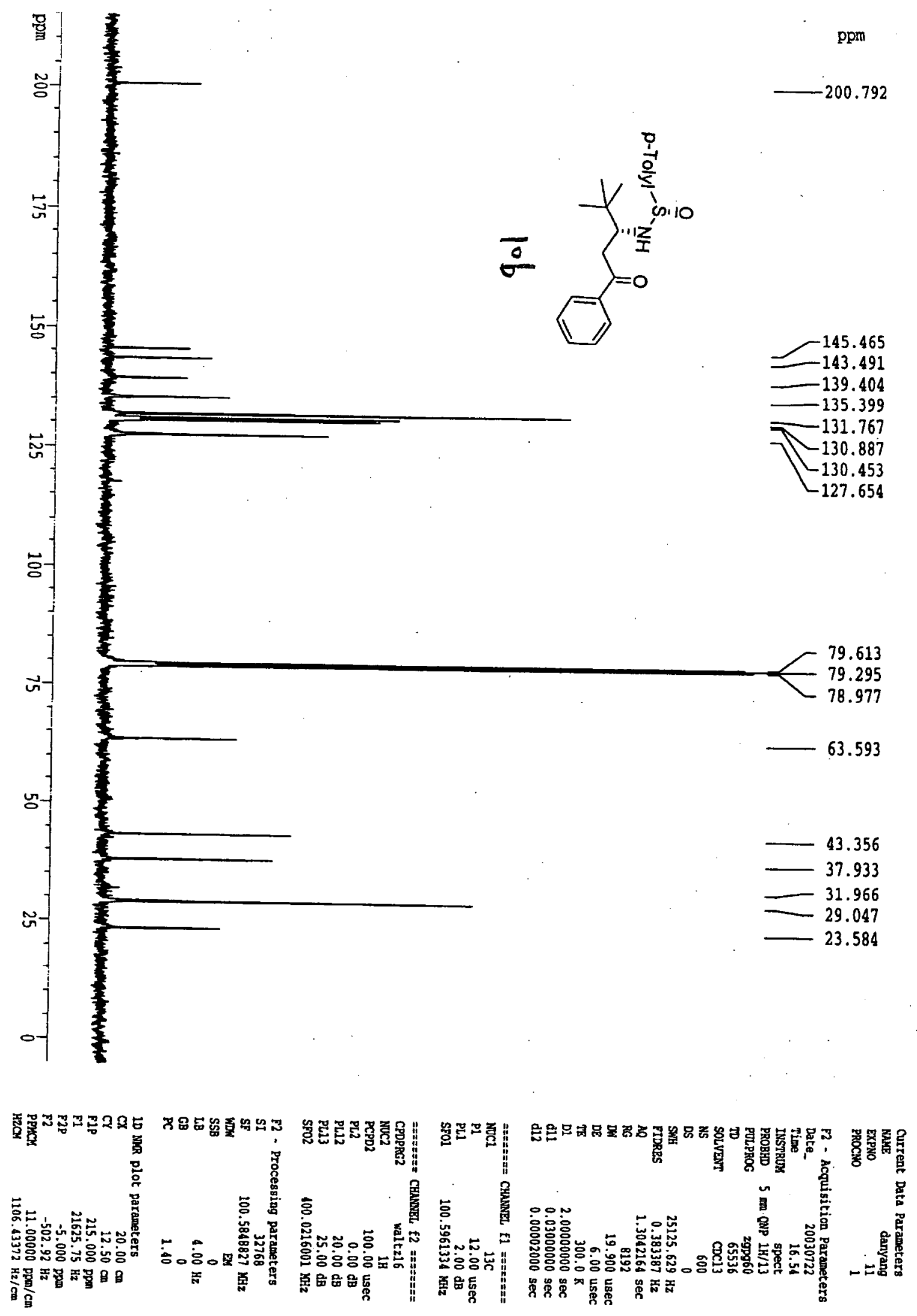
(C) 2005 American Chemical Society, J. Org. Chem., Davis jo0402780 Supporting Info Page 27

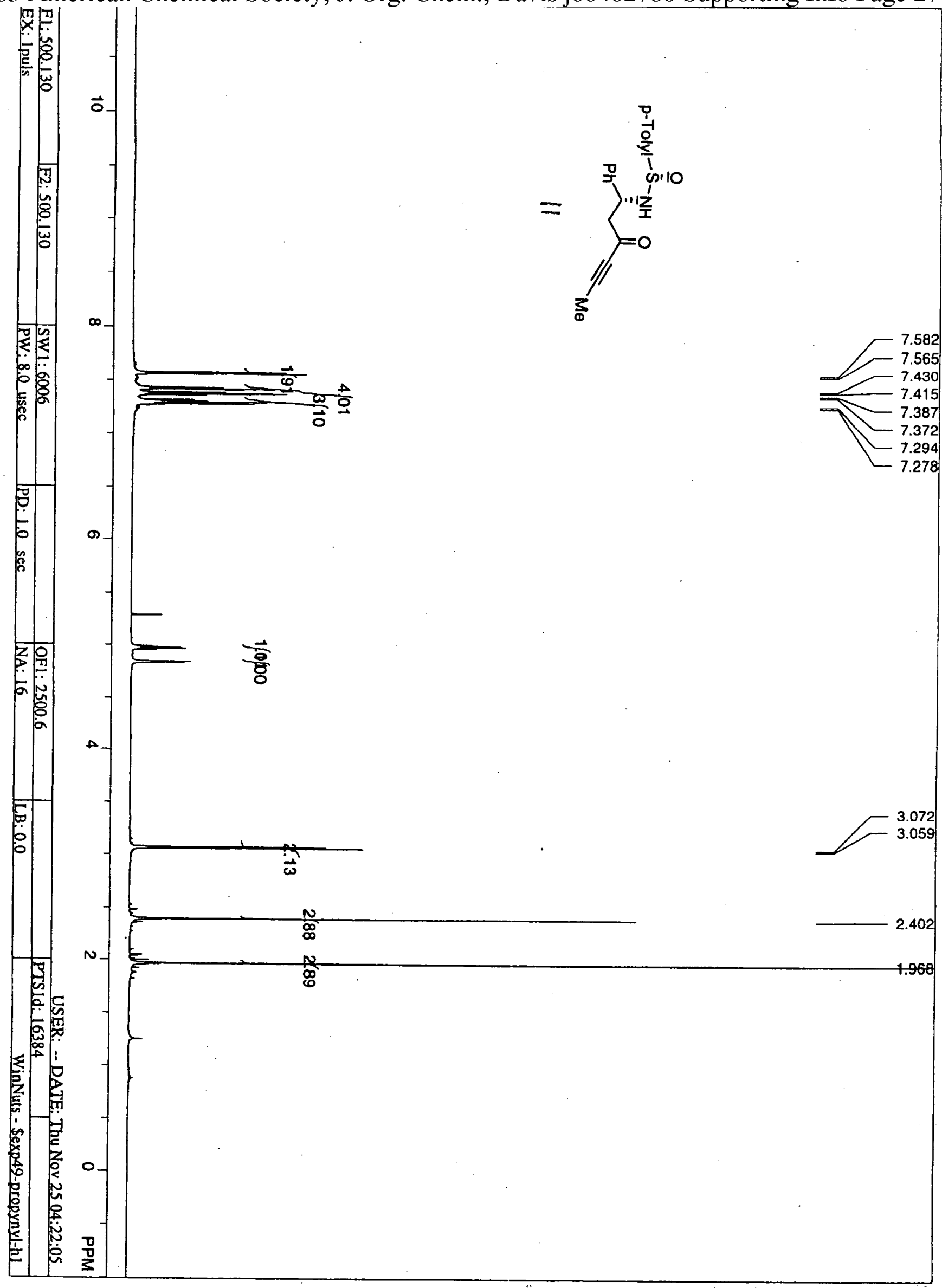


(C) 2005 American Chemical Society, J. Org. Chem., Davis jo0402780 Supporting Info Page 28

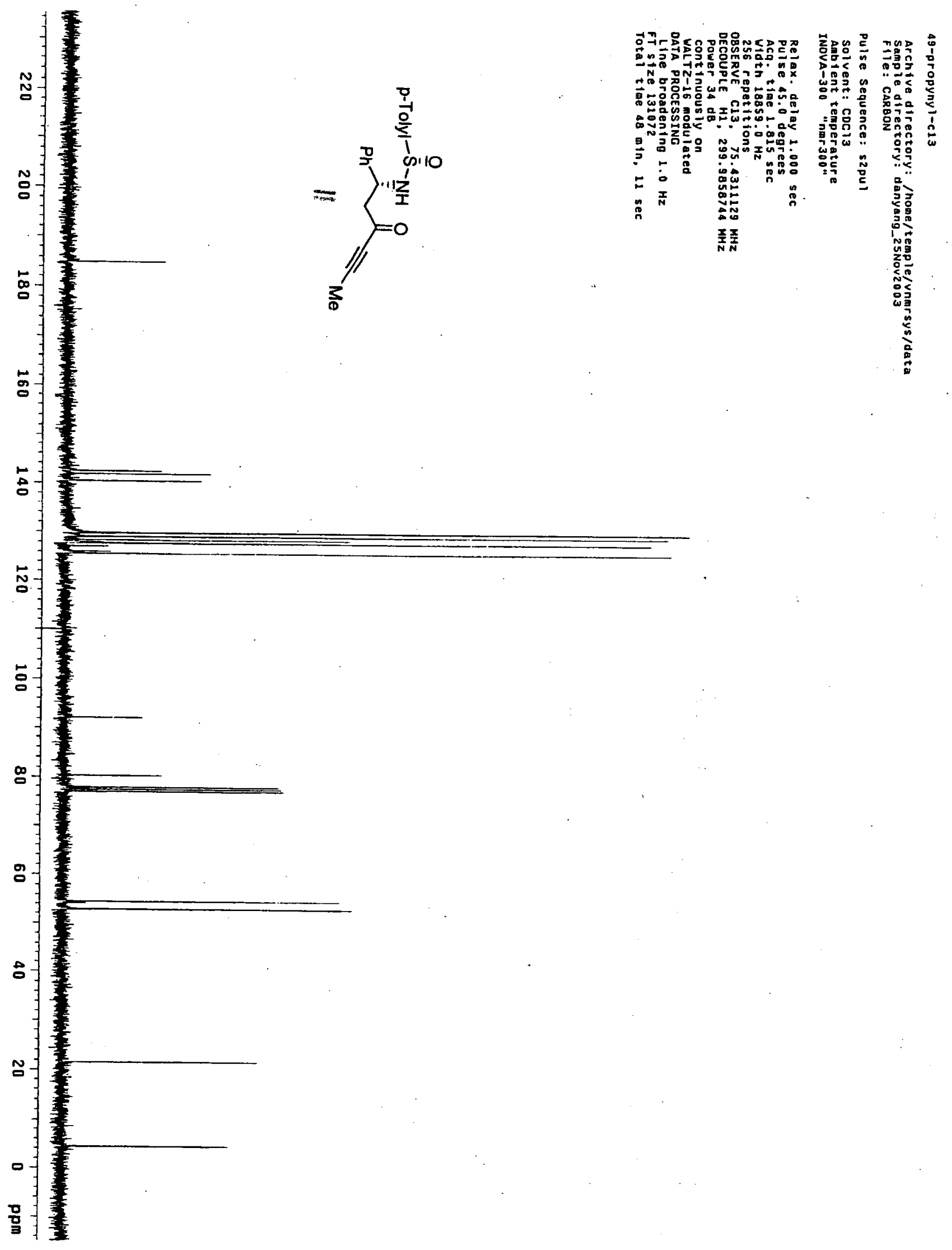


(1) 2005 Ämerican Chemical Society, J. Org. Chem., Davis jo0402780 Supporting Info Page 29

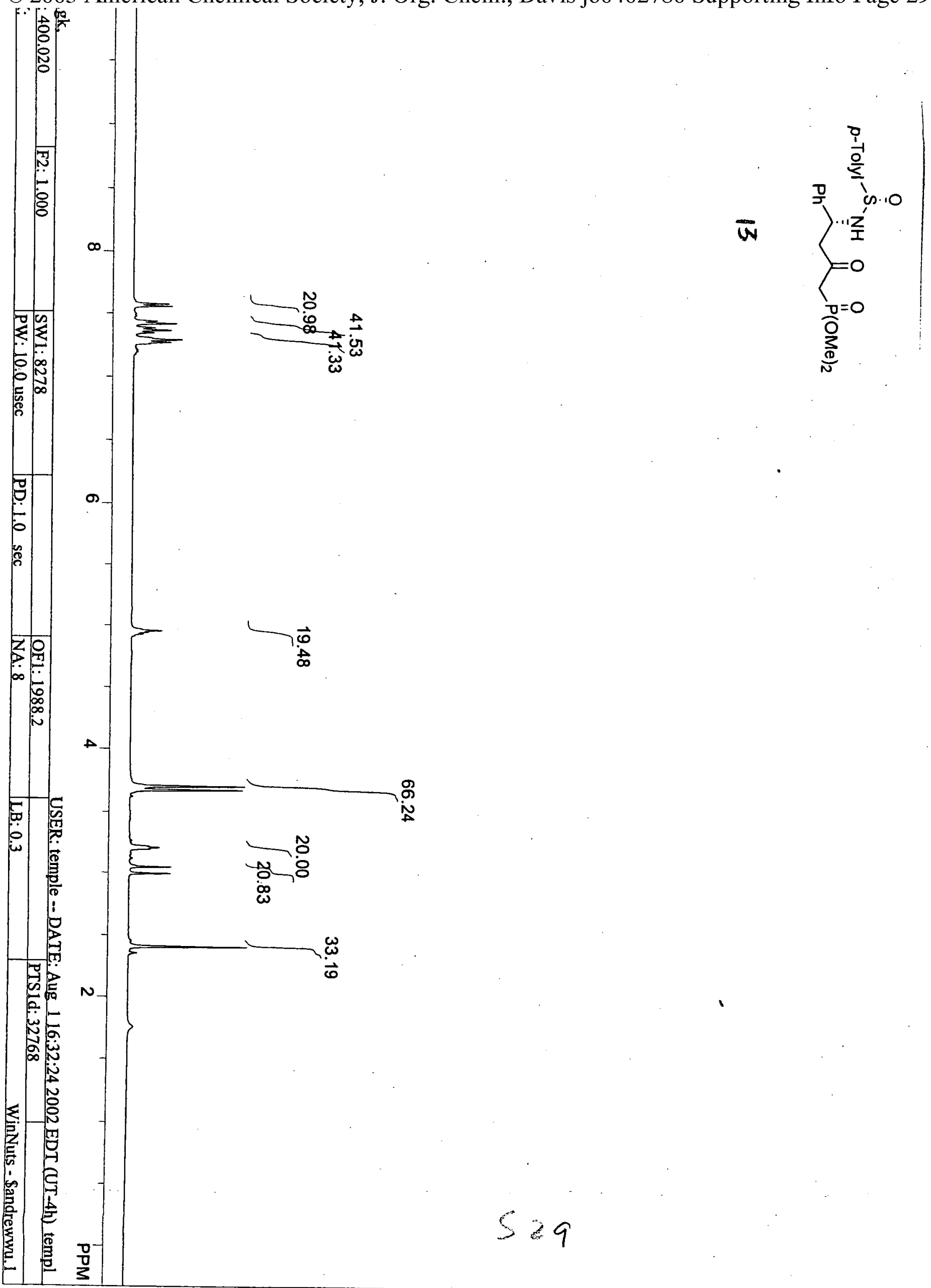


(C) 2005 American Chemical Society, J. Org. Chem., Davis jo0402780 Supporting Info Page 30

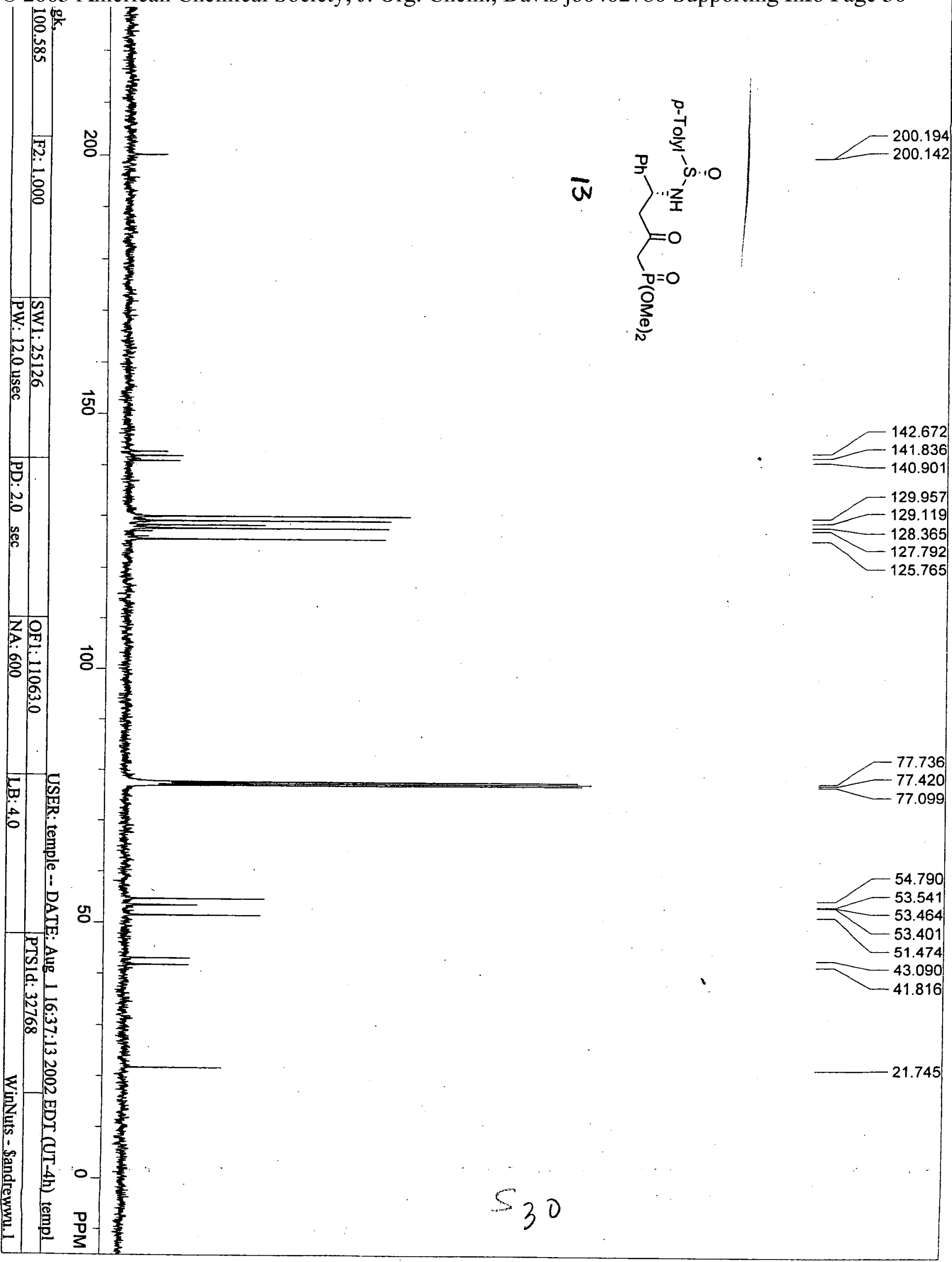


(C) 2005 American Chemical Society, J. Org. Chem., Davis jo0402780 Supporting Info Page 31

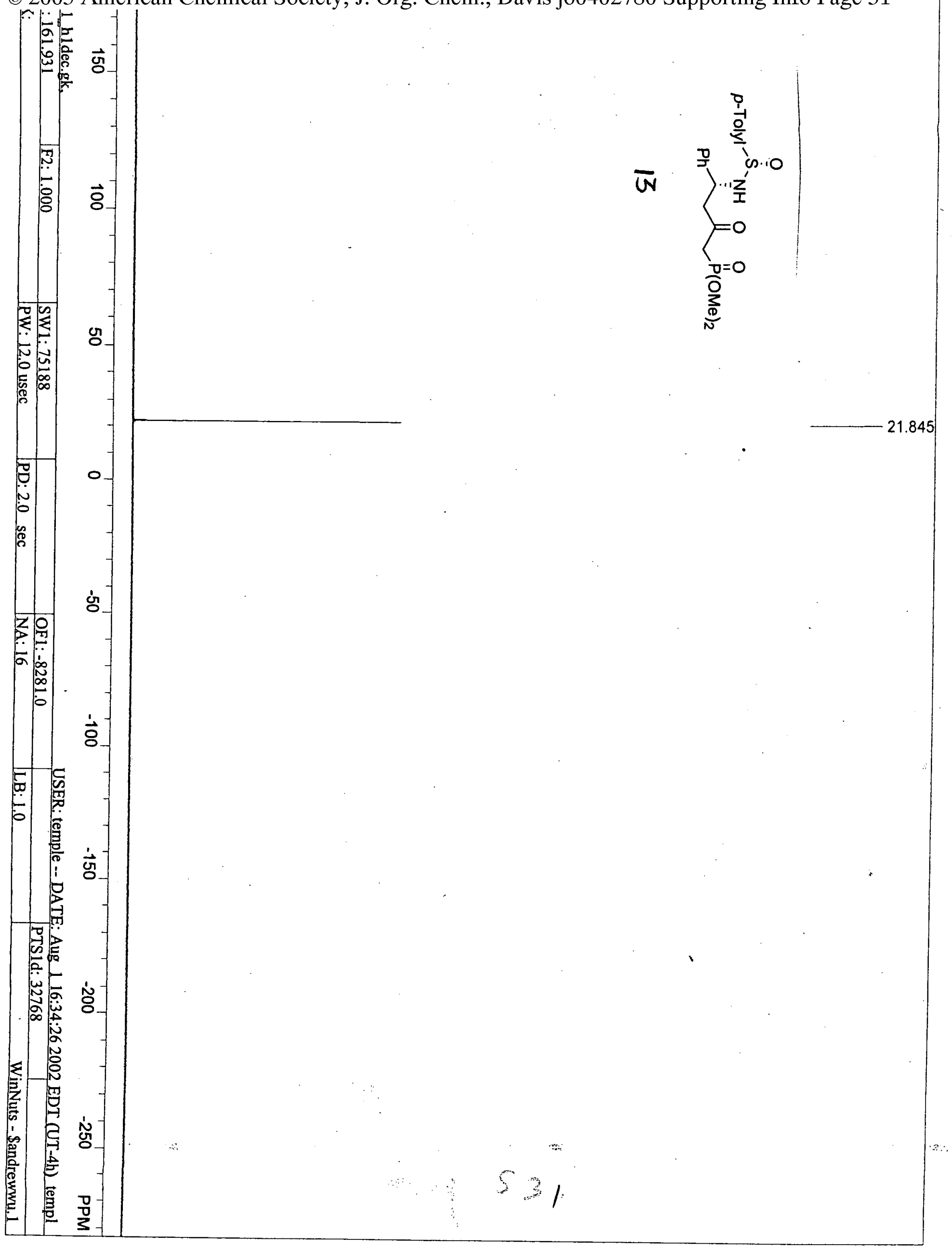


(c) 2005 American Chemical Society, J. Org. Chem., Davis jo0402780 Supporting Info Page 32

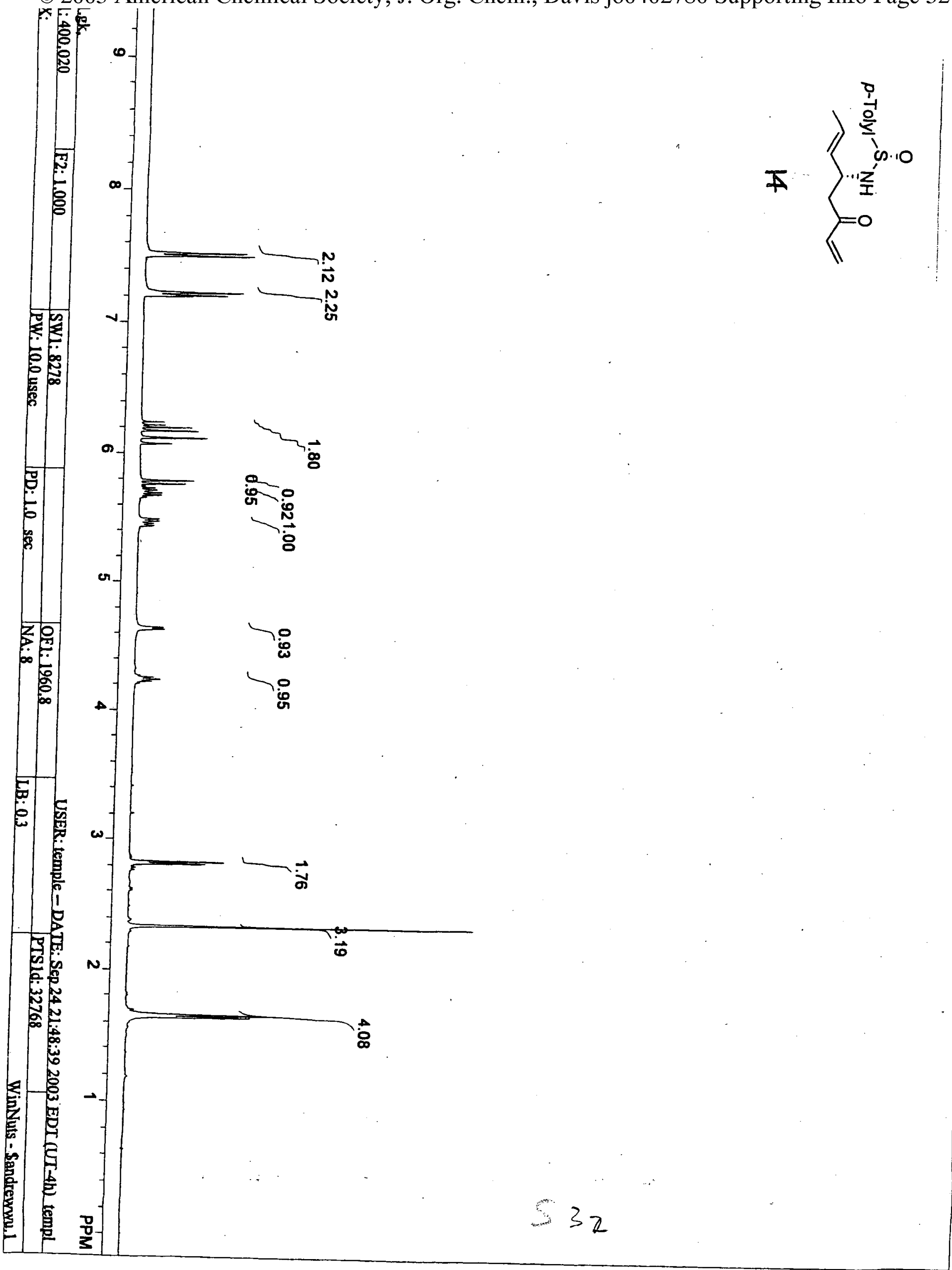


5. 2005 American Chemical Society, J. Org. Chem., Davis jo0402780 Supporting Info Page 33
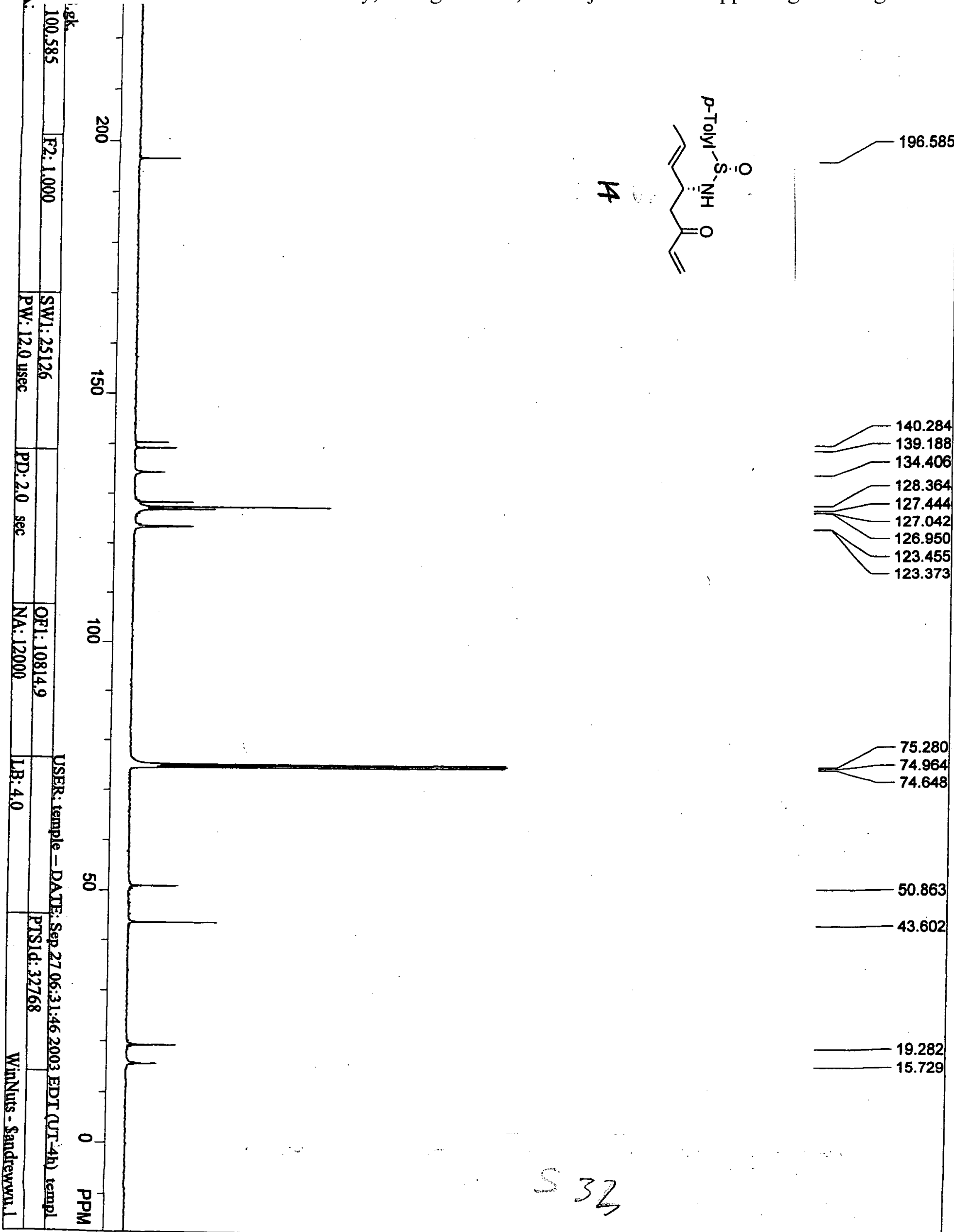
(3) 35
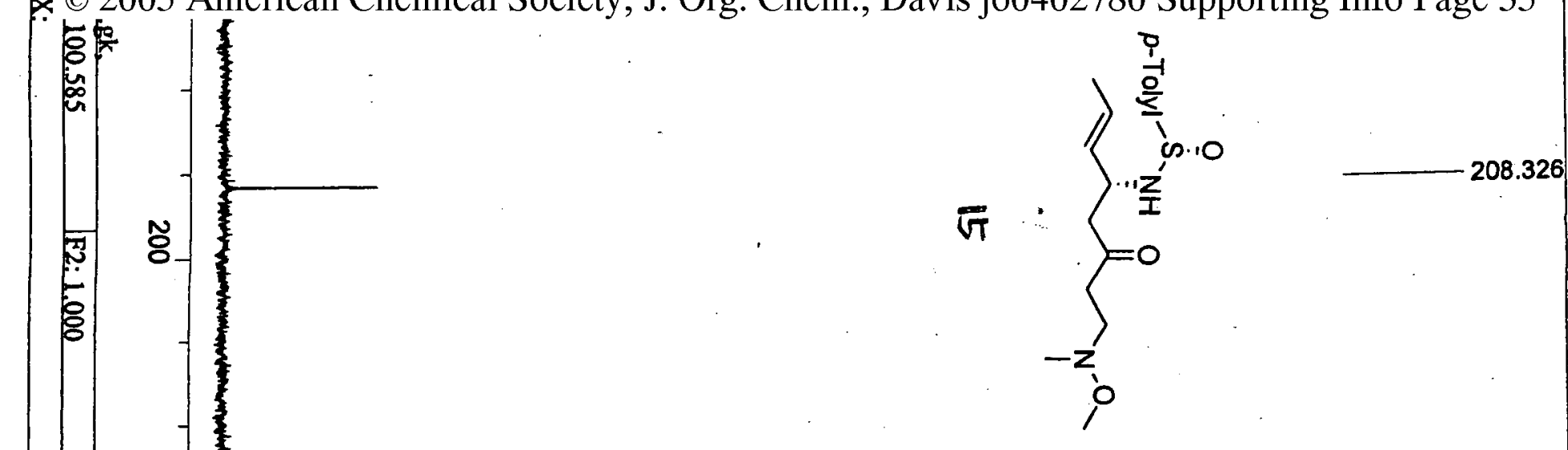

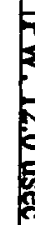

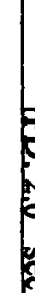

$\overrightarrow{5}$

胥

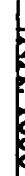

용
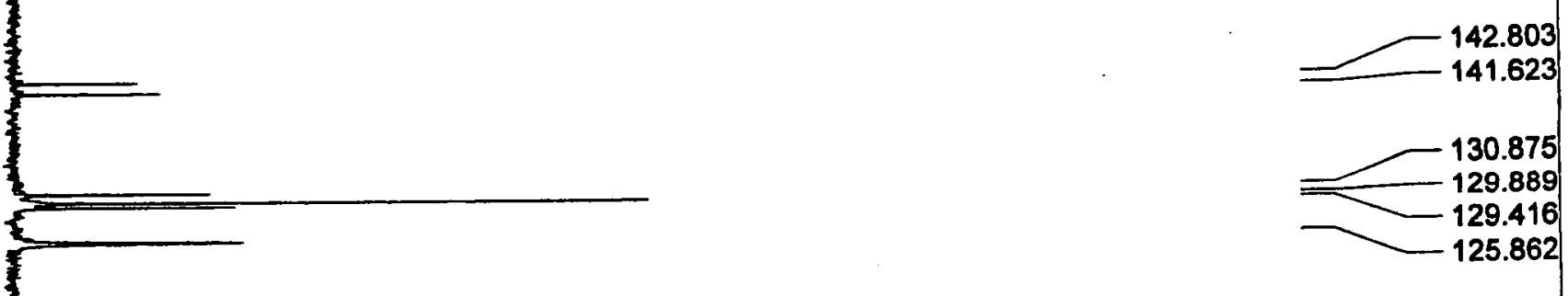

遂

8

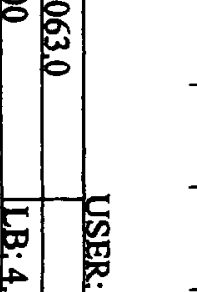

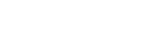

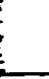

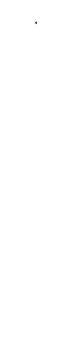

or

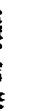
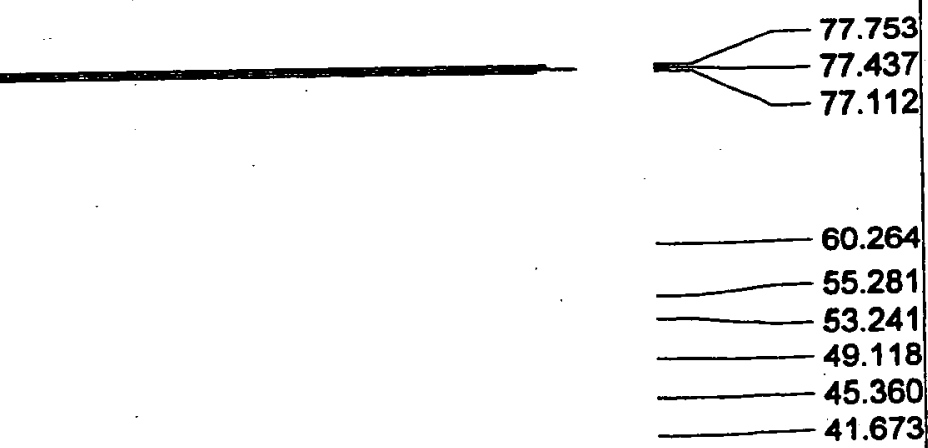

?

ب.

⿵人⿱亠乂

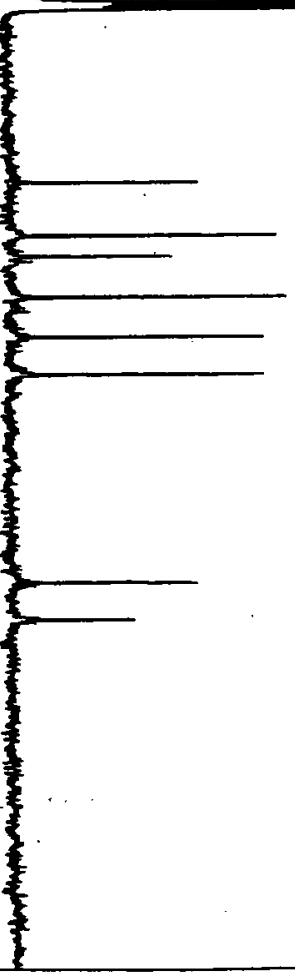


(c) 2005 American Chemical Society, J. Org. Chem., Davis jo0402780 Supporting Info Page 36

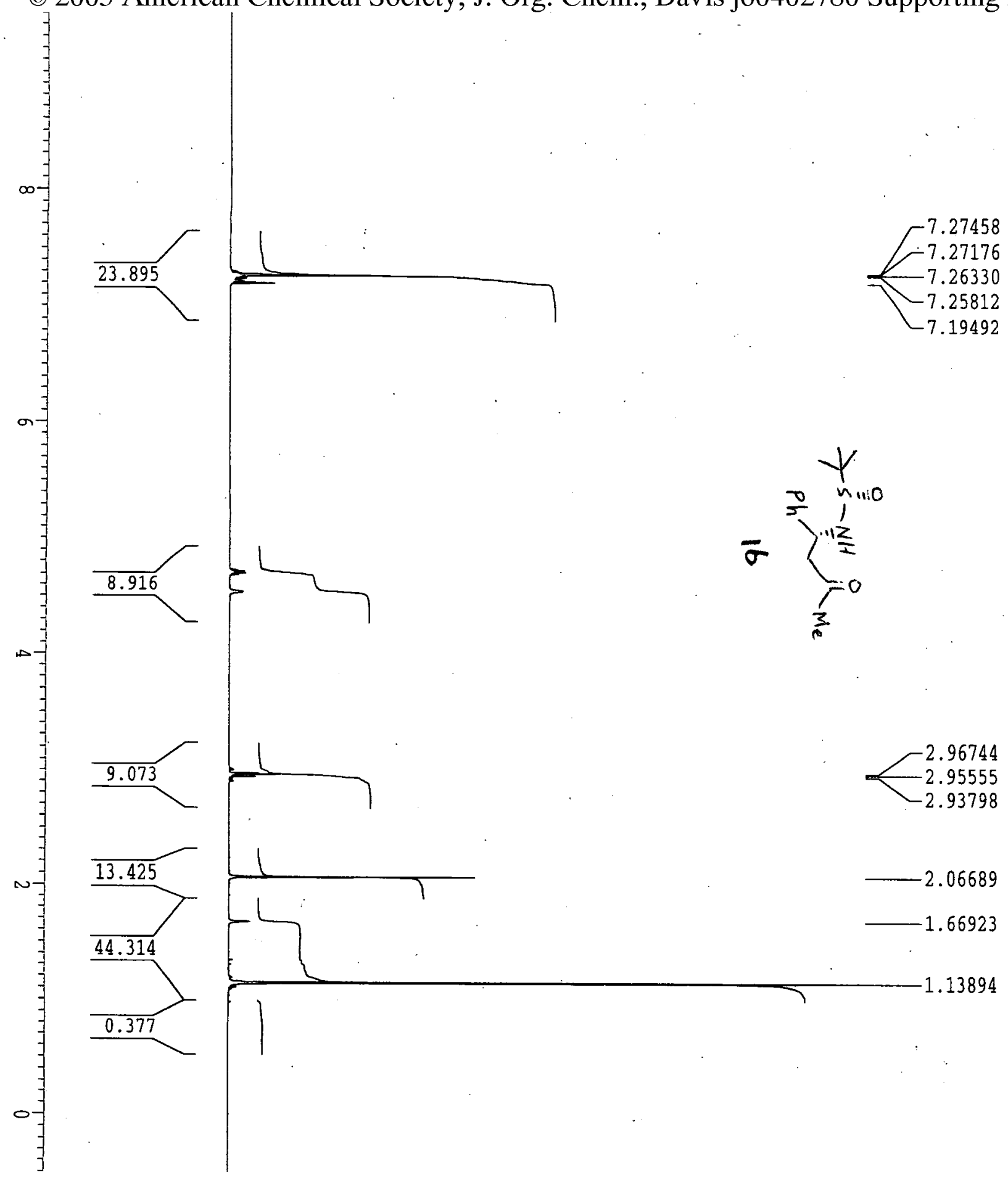

吉心

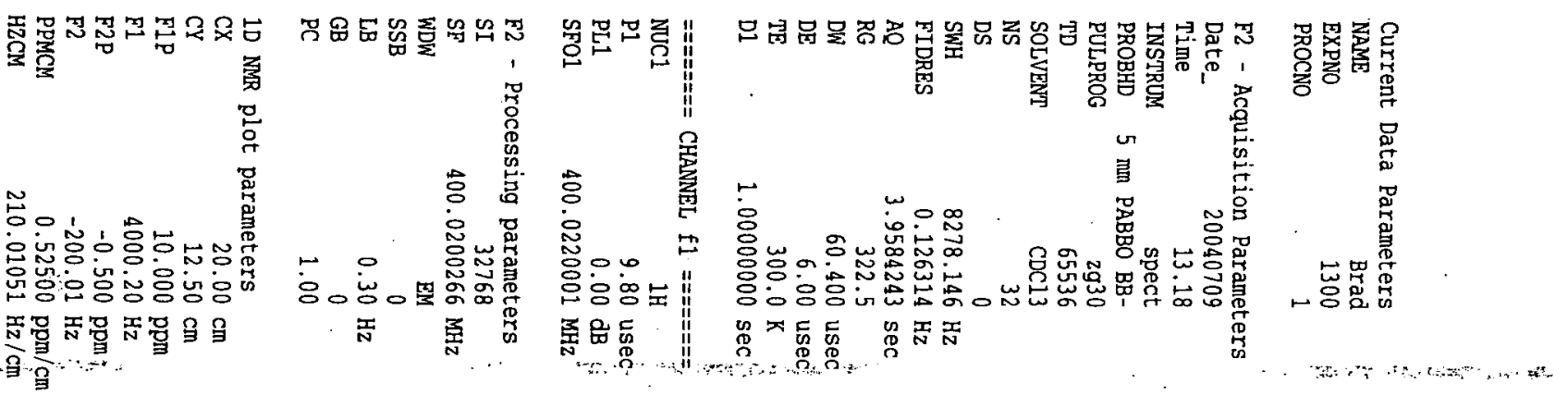

$$
536
$$


